\title{
Wa Ve phenomena

Global well-posedness and exponential stability for heterogeneous anisotropic Maxwell's equations under a nonlinear boundary feedback with delay

Andrii Anikushyn, Michael Pokojovy

CRC Preprint 2018/10, June 2018

\section{KARLSRUHE INSTITUTE OF TECHNOLOGY}

CRC 1173

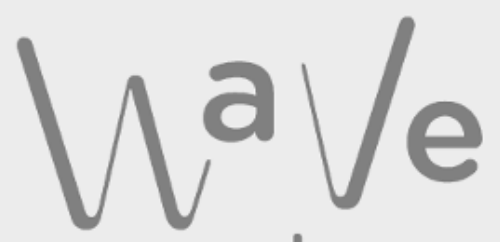

phenomena 


\section{Participating universities}

EBERHARD KARLS

UNIVERSITATT TUBINGEN

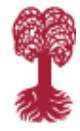

Funded by

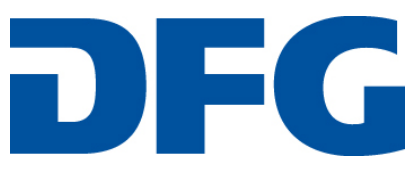

ISSN 2365-662X 


\title{
Global Well-Posedness and Exponential Stability for Heterogeneous Anisotropic Maxwell's Equations under a Nonlinear Boundary Feedback with Delay
}

\author{
Andrii Anikushyn* Michael Pokojovy ${ }^{\dagger}$
}

June 29, 2018

\begin{abstract}
We consider an initial-boundary value problem for the Maxwell's system in a bounded domain with a linear inhomogeneous anisotropic instantaneous material law subject to a nonlinear Silver-Müller-type boundary feedback mechanism incorporating both an instantaneous damping and a time-localized delay effect. By proving the maximal monotonicity property of the underlying nonlinear generator, we establish the global well-posedness in an appropriate Hilbert space. Further, under suitable assumptions and geometric conditions, we show the system is exponentially stable.
\end{abstract}

Key words: Maxwell's equations, nonlinear boundary feedback, instantaneous damping, time-localized delay, well-posedness, exponential stability

MSC (2010): $\quad$ Primary 35Q61, 35L50, 35L60, 35B40, 39B99

Secondary 35A01, 35A02, 35B37, 93C20, 93C23

\section{Introduction}

Consider the macroscopic formulation of Maxwell's equations in a bounded domain $G \subset \mathbb{R}^{3}$ with $\boldsymbol{\nu}: \Gamma \rightarrow \mathbb{R}^{3}$ standing for the outer normal vector to its smooth boundary $\Gamma:=\partial G$ and the functions $\mathbf{E}, \mathbf{D}, \mathbf{H}, \mathbf{B}:[0, \infty) \times G \rightarrow \mathbb{R}^{3}$ denoting the electric, displacement, magnetic and magnetizing fields, respectively. With $\rho:[0, \infty) \times G \rightarrow \mathbb{R}$ representing the electric charge density, Gauss' law along with Gauss' law for magnetism yield

$$
\operatorname{div} \mathbf{D}=\rho \quad \text { and } \quad \operatorname{div} \mathbf{B}=0 \quad \text { in }(0, \infty) \times G,
$$

while Faraday's law of induction and Ampère's circuital law mandate

$$
\partial_{t} \mathbf{D}=\operatorname{curl} \mathbf{H}-\mathbf{J} \quad \text { and } \quad \partial_{t} \mathbf{B}=-\operatorname{curl} \mathbf{E} \quad \text { in }(0, \infty) \times G .
$$

Typically, $\mathbf{J}:[0, \infty) \times G \rightarrow \mathbb{R}^{3}$ is a (given) total current density.

Since the system $(1.1)-(1.2)$ is underdetermined, two more equations relating the four unknown vector fields $\mathbf{E}, \mathbf{D}, \mathbf{H}, \mathbf{B}$ need to be postulated. Letting $\varepsilon, \boldsymbol{\mu}: G \rightarrow \mathbb{R}^{3 \times 3}$ be symmetric,

\footnotetext{
${ }^{*}$ Department of Computer Sciences and Cybernetics, Taras Shevcheno National University of Kyiv anik_andrii@univ.kiev.ua

${ }^{\dagger}$ Department of Mathematical Sciences, The University of Texas at El Paso, El Paso, TX mpokojovy@utep.edu
} 
uniformly positive definite matrix-valued permittivity and permeability tensor fields, the instantaneous anisotropic material laws read as

$$
\mathbf{D}=\varepsilon \mathbf{E} \quad \text { and } \quad \mathbf{B}=\boldsymbol{\mu} \mathbf{H} .
$$

Combining Equations (1.1)-1.3, we arrive at

$$
\begin{aligned}
& \partial_{t}(\varepsilon \mathbf{E})=\operatorname{curl} \mathbf{H}-\mathbf{J}, \quad \operatorname{div}(\varepsilon \mathbf{E})=\rho \quad \text { in }(0, \infty) \times G, \\
& \partial_{t}(\boldsymbol{\mu H})=-\operatorname{curl} \mathbf{E}, \quad \operatorname{div}(\boldsymbol{\mu H})=0 \quad \text { in }(0, \infty) \times G \text {. }
\end{aligned}
$$

Various boundary conditions for Equations (1.4)-(1.5) are known in the literature. Eller et al. [9] considered the nonlinear version

$$
\mathbf{H} \times \boldsymbol{\nu}+\mathbf{g}(\mathbf{E} \times \boldsymbol{\nu}) \times \boldsymbol{\nu}=\mathbf{0} \quad \text { on }(0, \infty) \times \Gamma
$$

of the classical Silver-Müller boundary condition

$$
\mathbf{H} \times \boldsymbol{\nu}+\kappa \cdot(\mathbf{E} \times \boldsymbol{\nu}) \times \boldsymbol{\nu}=\mathbf{0} \quad \text { on }(0, \infty) \times \Gamma .
$$

Here, $\mathbf{g}: \mathbb{R}^{3} \rightarrow \mathbb{R}^{3}$ is a smooth function with $\mathbf{g}(\mathbf{0})=\mathbf{0}$ and $\kappa>0$ is a constant. Equations 1.6 and (1.7) model scattering of electromagnetic waves by an obstacle $G$ under the assumption that the waves cannot penetrate the obstacle too deeply [3, p. 20]. The Silver-Müller boundary condition (1.7) arises as a first-order approximation to the so-called transparent boundary condition but, despite of being dissipative, allows for reflections back into the domain $G$ [8, p. 136].

In the present paper, we modify the nonlinear feedback-type boundary condition (1.6) by incorporating a nonlinear time-localized delay effect:

$$
\mathbf{H}(t, \cdot) \times \boldsymbol{\nu}+\gamma_{1} \mathbf{g}(\mathbf{E}(t, \cdot) \times \boldsymbol{\nu}) \times \boldsymbol{\nu}+\gamma_{2} \mathbf{g}(\mathbf{E}(t-\tau, \cdot) \times \boldsymbol{\nu}) \times \boldsymbol{\nu}=\mathbf{0} \quad \text { on }(0, \infty) \times \Gamma
$$

with a delay parameter $\tau>0$ and appropriate constants $\gamma_{1}, \gamma_{2}>0$. Viewing the instantaneous Silver-Müller boundary conditions (1.6) and (1.7) as a feedback boundary control, the latter being a common stabilization instrument widely used in engineering, an extra delay term in Equation (1.8) becomes indispensable to adequately account for time retardations, which inevitably arise due a time lag in the interaction between a sensor measuring $\mathbf{E} \times \boldsymbol{\nu}$ and the actuator updating $\mathbf{H} \times \boldsymbol{\nu}$ on the boundary $\Gamma$.

Pulling Equations (1.4)-1.5), (1.8) together, we arrive at

$$
\begin{array}{rlrl}
\partial_{t}(\varepsilon \mathbf{E})=\operatorname{curl} \mathbf{H}-\mathbf{J}, & \operatorname{div}(\varepsilon \mathbf{E})=\rho & & \text { in }(0, \infty) \times G, \\
\partial_{t}(\boldsymbol{\mu} \mathbf{H})=-\operatorname{curl} \mathbf{E}, & \operatorname{div}(\boldsymbol{\mu H})=0 & & \text { in }(0, \infty) \times G, \\
\mathbf{H}(t, \cdot) \times \boldsymbol{\nu}+\gamma_{1} \mathbf{g}(\mathbf{E}(t, \cdot) \times \boldsymbol{\nu}) \times \boldsymbol{\nu}+\gamma_{2} \mathbf{g}(\mathbf{E}(t-\tau, \cdot) \times \boldsymbol{\nu}) \times \boldsymbol{\nu}=\mathbf{0} & & \text { on }(0, \infty) \times \Gamma .
\end{array}
$$

In the following, let $\mathbf{J} \equiv 0$ and $\rho \equiv 0$. This corresponds to the case both electrical sources and resistance effects are absent. While not affecting the well-posedness results to follow, compared to the case of electrical resistance, i.e., $\mathbf{J}=\boldsymbol{\sigma}(\mathbf{E}, \mathbf{H}) \mathbf{E}$ as mandated by the Ohm's law, the condition $\mathbf{J} \equiv 0$ reduces the overall amount of damping in the system making the stability analysis more challenging. Adding the usual initial conditions, we arrive at the system

$$
\begin{aligned}
\partial_{t}(\varepsilon \mathbf{E})=\operatorname{curl} \mathbf{H}, \quad \operatorname{div}(\varepsilon \mathbf{E})=0 & & \text { in }(0, \infty) \times G, \\
\partial_{t}(\boldsymbol{\mu} \mathbf{H})=-\operatorname{curl} \mathbf{E}, \quad \operatorname{div}(\boldsymbol{\mu} \mathbf{H})=0 & & \text { in }(0, \infty) \times G, \\
\mathbf{H}(t, \cdot) \times \boldsymbol{\nu}+\gamma_{1} \mathbf{g}(\mathbf{E}(t, \cdot) \times \boldsymbol{\nu}) \times \boldsymbol{\nu}+\gamma_{2} \mathbf{g}(\mathbf{E}(t-\tau, \cdot) \times \boldsymbol{\nu}) \times \boldsymbol{\nu}=\mathbf{0} & & \text { on }(0, \infty) \times \Gamma,
\end{aligned}
$$




$$
\begin{aligned}
& \mathbf{E}(0, \cdot)=\mathbf{E}^{0}, \quad \mathbf{H}(0, \cdot)=\mathbf{H}^{0} \quad \text { in } G, \\
& \mathbf{E}(-\tau \cdot, \cdot) \times \boldsymbol{\nu}=\boldsymbol{\Phi}^{0} \quad \text { in }(0,1) \times \Gamma .
\end{aligned}
$$

Partial (not mentioning ordinary!) differential equations (PDEs) have widely been studied in the literature. Time-delays along with other types of time-nonlocalities such as memory effects, etc., can typically enter a PDE in one of the two ways - either through a time-nonlocal material law [11, 13] or a time-delayed feedback mechanism (so-called "closed-loop control") [4, 5, 18, 23, 25], etc. Whereas time-delayed material laws mostly lead to ill-posedness [13, the effect of time-delay in feedback mechanisms can range from a "mere" reduction of the decay rate to destabilization to even ill-posedness. We refer the reader to the famous Datko's example [4, which illustrates the later dichotomy. Our goal is to investigate the impact of the nonlinear boundary delay feedback from Equation (1.11) on system (1.9) - 1.13). Before proceeding with our study, we first give a short literature review. In our brief review below, we restrict ourselves to instantaneous material laws but discuss both instantaneous and nonlocal boundary conditions.

As stated earlier, various boundary conditions for Equations $1.4-(1.5)$ have been studied in the literature. Eller et al. [9] examined the problem of stabilizing Maxwell's equations (1.9)-(1.10) subject to boundary condition

$$
\mathbf{H} \times \boldsymbol{\nu}+g(\mathbf{E} \times \boldsymbol{\nu}) \times \boldsymbol{\nu}=\mathbf{0} \text { on }(0, \infty) \times \Gamma .
$$

The (scalar) $\varepsilon$ and $\mu$ were assumed real positive fields and $g(\cdot)$ a continuous mapping satisfying certain monotonicity and boundness conditions. To prove the well-posedness, monotone operator theory and nonlinear semigroup theory were used, while the exponential stability - both in the linear and the nonlinear cases - was shown via exact controllability established using multiplier techniques.

Similar investigations were performed by Eller [6] who studied Equations $1.9-1.10$ with the boundary conditions

$$
\boldsymbol{\nu} \times \mathbf{E}=\mathbf{0}, \quad \boldsymbol{\nu} \cdot(\boldsymbol{\mu H})=0 \text { on }(0, T) \times \Gamma .
$$

Assuming the star-shapedness of $G$ and exploiting the method of multipliers, a boundary observability inequality was proved. Certain results on the boundary regularity of classical solutions to Maxwell's equations

$$
\varepsilon \partial_{t} \mathbf{E}-\operatorname{curl} \mathbf{H}+\sigma \mathbf{E}=\mathbf{J}, \quad \boldsymbol{\mu} \partial_{t} \mathbf{H}+\operatorname{curl} \mathbf{E}=\mathbf{0} \text { in }(0, T) \times G,
$$

complemented by the boundary and initial conditions

$$
\boldsymbol{\nu} \times \mathbf{E}=\mathbf{0} \text { on }(0, T) \times \Gamma, \quad \mathbf{E}(0)=\mathbf{E}^{0}, \quad \mathbf{H}(0)=\mathbf{H}^{0} \text { in } G
$$

were obtained by the same author in [7].

Lagnese [16] studied the exact boundary controllability of Maxwell's equations

$$
\begin{aligned}
\partial_{t} \mathbf{E}-\operatorname{curl}\left(\varepsilon^{-1} \mathbf{H}\right)=\mathbf{0}, \quad \partial_{t} \mathbf{H}+\operatorname{curl}\left(\mu^{-1} \mathbf{E}\right) & =\mathbf{0} \text { in }(0, \infty) \times G, \\
\operatorname{div} \mathbf{E}=\operatorname{div} \mathbf{H} & =\mathbf{0} \text { in }(0, \infty) \times G, \\
\mathbf{E}(0)=\mathbf{E}^{0}, \quad \mathbf{H}(0) & =\mathbf{H}^{0} \text { in } G
\end{aligned}
$$

subject to boundary condition

$$
\boldsymbol{\nu} \times \mathbf{H}=-\mathbf{J} \text { on } \Gamma \times(0, \infty)
$$


Here, the current density $\mathbf{J}$ plays the role of a distributed open-loop control. The electric permittivity $\varepsilon$ and magnetic permeability $\mu$ were assumed constant, while the region $G$ was selected to be starshaped with respect to some point.

Nicaise [19] investigated the exact controllability of isotropic Maxwell's equations 1.9$]-(1.10)$ with the boundary conditions

$$
\begin{aligned}
& \mathbf{H} \times \boldsymbol{\nu}=\mathbf{J} \text { on }(0, T) \times \Gamma_{0}, \\
& \mathbf{H} \times \boldsymbol{\nu}=\mathbf{0} \text { on }(0, T) \times\left(\Gamma \backslash \Gamma_{0}\right), \\
& \mathbf{E} \times \boldsymbol{\nu}=\mathbf{0} \text { on }(0, T) \times \Gamma
\end{aligned}
$$

via a boundary control $\mathbf{J}$. Here, $\Gamma_{0}$ is a non-empty, relatively open subset of $\Gamma$.

Eller \& Masters [10] later used multiplier techniques to prove the exact controllability for Equations (1.14)-(1.16) via of the boundary control

$$
\boldsymbol{\nu} \times\left(\varepsilon^{-1} \mathbf{H}\right)=\mathbf{J} \text { on }(0, T) \times \Gamma
$$

for nonhomogeneous $\mu, \varepsilon$ without any star-shapedness assumptions.

Krigman [15] studied a similar problem for the system

$$
\begin{aligned}
\varepsilon \partial_{t} \mathbf{E}-\operatorname{curl}(\mathbf{H})+\sigma \mathbf{E}=\mathbf{0}, \quad \mu \partial_{t} \mathbf{H}+\operatorname{curl}(\mathbf{E})=\mathbf{0} \text { in }(0, \infty) \times G \\
\operatorname{div}(\varepsilon \mathbf{E})=0, \quad \operatorname{div}(\boldsymbol{\mu H})=0 \text { in }(0, \infty) \times G
\end{aligned}
$$

with the initial conditions $(1.16)$ and boundary condition 1.17 with no star-shapedness assumptions.

Zhou [27] investigated the exact controllability under the action of a distributed control $\mathbf{u}$

$$
\begin{aligned}
\partial_{t} \mathbf{E}-\operatorname{curl}(\mathbf{H}) & =\chi_{G}(\mathbf{x}) \mathbf{u} \text { in }(0, \infty) \times G, \\
\partial_{t} \mathbf{H}+\operatorname{curl}(\mathbf{E}) & =\mathbf{0} \text { in }(0, \infty) \times G, \\
\operatorname{div} \mathbf{H}=\operatorname{div} \mathbf{E} & =0 \text { in }(0, \infty) \times G, \\
\mathbf{E} \times \boldsymbol{\nu} & =\mathbf{0} \text { on }(0, \infty) \times G, \\
\mathbf{E}(0)=\mathbf{E}^{0}, \quad \mathbf{H}(0) & =\mathbf{H}^{0} \text { in } G,
\end{aligned}
$$

where $\chi_{G}(\cdot)$ is the indicator function of a set $\omega \subset G$. This result was further extended by Zhang [26] to time-dependent $\omega$ 's using multiplier techniques.

A series of important results were obtained by Nicaise \& Pignotti. In [20], under monotonicity and boundedness assumptions on $g(\cdot)$, the authors considered a stabilization problem for Maxwell's equations

$$
\begin{aligned}
\partial_{t} \mathbf{E}-\operatorname{curl}(\lambda \mathbf{H}) & =\mathbf{0} \text { in }(0, \infty) \times G, \\
\partial_{t} \mathbf{H}+\operatorname{curl}(\boldsymbol{\mu} \mathbf{E}) & =\mathbf{0} \text { in }(0, \infty) \times G, \\
\operatorname{div} \mathbf{E}=\operatorname{div} \mathbf{H}=0 \operatorname{in}(0, \infty) \times G, & \mathbf{E}(0)=\mathbf{E}^{0}, \quad \mathbf{H}(0)=\mathbf{H}^{0} \text { in } G
\end{aligned}
$$

with space-time variable (scalar) coefficients $\boldsymbol{\mu}=\boldsymbol{\mu}(\mathbf{x}, t), \lambda=\lambda(\mathbf{x}, t)$ and a nonlinear Silver-Müller boundary condition

$$
g(\mathbf{x}, \mathbf{E} \times \boldsymbol{\nu})+\mathbf{H} \times \boldsymbol{\nu}=\mathbf{0} \text { on }(0, \infty) \times \Gamma .
$$


Another article 21] by the same authors was dedicated to the problem of stabilization of Maxwell's equations via a distributed feedback arising from the linear Ohm's law:

$$
\begin{aligned}
\partial_{t} \mathbf{E}-\operatorname{curl}(\lambda \mathbf{H})+\sigma \mathbf{E} & =\mathbf{0} \text { in }(0, \infty) \times G, \\
\partial_{t} \mathbf{H}+\operatorname{curl}(\boldsymbol{\mu} \mathbf{E}) & =\mathbf{0} \text { in }(0, \infty) \times G, \\
\operatorname{div} \mathbf{H} & =0 \text { in }(0, \infty) \times G, \\
\mathbf{E} \times \boldsymbol{\nu}=\mathbf{0}, & \mathbf{H} \cdot \boldsymbol{\nu}=0 \text { on }(0, \infty) \times \Gamma, \\
\mathbf{E}(0)=\mathbf{E}^{0}, & \mathbf{H}(0)=\mathbf{H}^{0} \text { in } G .
\end{aligned}
$$

The method of multipliers was used to establish an observability estimate in the paper. Same authors 222 also obtained an observability estimate for the standard isotropic homogeneous Maxwell's system (1.18)-1.21) subject to boundary conditions (1.25).

The impact of boundary conditions that include tangetial components were studied by numerous authors. Kapitonov [12] considered Equations (1.18) - 1.21) in $(0, T) \times G$ with dissipative boundary conditions

$$
\boldsymbol{\nu} \times \mathbf{E}-\alpha(\cdot) \mathbf{H}_{\tau}=\mathbf{0},
$$

where $\alpha(\cdot)$ is a continuously differentiable function on $\Gamma$ with $\operatorname{Re} \alpha>0$. Here and in the sequel,

$$
\mathbf{H}_{\tau}:=\mathbf{H}-(\mathbf{H} \times \boldsymbol{\nu}) \mathbf{H}
$$

denotes the tangential component of $\mathbf{H}$. Using the semigroup approach to investigate the wellposedness, the author further utilized geometrical properties of the domain to obtain results on exact boundary controllability of the solution to $1.18-(1.21)$ in $(0, T) \times G$ with boundary condition

$$
\boldsymbol{\nu} \times \mathbf{E}-\left.\mathrm{i} a(\mathbf{x}) \mathbf{H}_{\tau}\right|_{\Gamma}=\mathbf{p}(t, \mathbf{x}),
$$

where $a(\mathbf{x})$ is a continuously differentiable scalar function on $\Gamma$. Cagnol and Eller [2] studied a similar problem for anisotropic Maxwell's equations with the so-called "absorbing boundary" condition

$$
\boldsymbol{\nu} \times \mathbf{E}-\alpha \mathbf{H}_{\tau}=\mathbf{g} \text { on }(0, T) \times \Gamma .
$$

Nonlocal boundary conditions are also known in the literature. Nibbi \& Polidoro [18] proved the exponential stability of 'Graffi'-type free energy associated with the isotropic Maxwell's equations subject to a memory-type boundary condition

$$
\mathbf{E}_{\tau}(t, \cdot)=\eta_{0} \mathbf{H}(t, \cdot) \times \boldsymbol{\nu}+\int_{0}^{\infty} \eta(s) \mathbf{H}(t-s, \cdot) \times \boldsymbol{\nu} \mathrm{d} s .
$$

In contrast, the impact of time-delayed boundary conditions from Equation (1.11) on Maxwell's equations has not been studied in the literature before. At the same time, such boundary conditions proved to be very interesting - both from theoretical in practical point of view - for other types of hyperbolic systems. For example, Nicaise \& Pignotti [23] investigated the stability of a delay wave equation subject to a time-delayed boundary feedback

$$
\begin{gathered}
u_{t t}(t, \mathbf{x})-\Delta u(t, \mathbf{x})=0 \text { for }(t, \mathbf{x}) \in(0, \infty) \times G, \\
u(t, \mathbf{x})=0 \text { for }(t, \mathbf{x}) \in(0, \infty) \times \Gamma_{0}, \\
\partial_{\nu} u(t, \mathbf{x})=-\mu_{1} u_{t}(t, \mathbf{x})-\mu_{2} u(t-\tau, \mathbf{x}) \text { for }(t, \mathbf{x}) \in(0, \infty) \times\left(\Gamma \backslash \Gamma_{0}\right), \\
u(0, \mathbf{x})=u_{0}(\mathbf{x}), \partial_{t} u(0, \mathbf{x})=u_{1}(\mathbf{x}) \text { for } \mathbf{x} \in G,
\end{gathered}
$$




$$
u_{t}(t-\tau, \mathbf{x})=f_{0}(t-\tau, \mathbf{x}) \text { for }(t, \mathbf{x}) \in(0, \tau) \times\left(\Gamma \backslash \Gamma_{0}\right) .
$$

Under suitable conditions on $\Gamma_{0}$, the initial-boundary-value problem was shown to possess a unique strong solution, which is exponentially stable given $\mu_{2}<\mu_{1}$.

The rest of the paper has the following outline. In Section 2, partial difference-differential Equations (1.9)-1.13) are transformed to an abstact nonlinear evolution equation on the extended phase space. By showing maximal monotonicity of the generator and exploiting the nonlinear semigroup theory, the well-posedness is proved. In Section 3, under a star-shapedness assumption on the domain $G$, the exponential stability of the system is shown by using standard Rellich's multipliers and auxiliary functions inspired by [13]. In the Appendix section, for the sake of completeness, a "folklore" method (which probably goes back to early works of I. Lasiecka) that establishes a connection between disipativity, an observability-through-damping inequality and exponential stability is formulated and proved.

\section{Well-Posedness}

Following [6], for a symmetric, positive definite matrix-valued $\boldsymbol{\alpha} \in L^{\infty}\left(G, \mathbb{R}^{3 \times 3}\right)$, we define the spaces

$$
\begin{aligned}
H(\operatorname{curl}, G) & :=\left\{\mathbf{u} \in\left(L^{2}(G)\right)^{3} \mid \operatorname{curl} \mathbf{u} \in\left(L^{2}(G)\right)^{3}\right\}, \\
H\left(\operatorname{div}_{\boldsymbol{\alpha}} 0, G\right) & :=\left\{\mathbf{u} \in\left(L^{2}(G)\right)^{3} \mid \operatorname{div}(\boldsymbol{\alpha} \mathbf{u})=0\right\}
\end{aligned}
$$

and introduce the Hilbert space

$$
\mathcal{H}:=H\left(\operatorname{div}_{\boldsymbol{\varepsilon}} 0, G\right) \times H\left(\operatorname{div}_{\boldsymbol{\mu}} 0, G\right)
$$

endowed with the inner product

$$
\left\langle(\mathbf{E}, \mathbf{H})^{T},(\tilde{\mathbf{E}}, \tilde{\mathbf{H}})^{T}\right\rangle_{\mathcal{H}}:=\int_{G} \varepsilon \mathbf{E} \cdot \tilde{\mathbf{E}} \mathrm{d} \mathbf{x}+\int_{G} \boldsymbol{\mu} \mathbf{H} \cdot \tilde{\mathbf{H}} \mathrm{d} \mathbf{x} .
$$

(The completeness follows from [16]).

Similar to [9], we formally define the operator

$$
\mathcal{A}:\left(\begin{array}{c}
\mathbf{E} \\
\mathbf{H}
\end{array}\right) \mapsto\left(\begin{array}{r}
-\varepsilon^{-1} \operatorname{curl} \mathbf{H} \\
\boldsymbol{\mu}^{-1} \operatorname{curl} \mathbf{E}
\end{array}\right) .
$$

Our goal is to transform Equations $1.9-1.13$ to an abstract Cauchy problem on the extended phase space (cf. [13, 23])

$$
\mathscr{H}:=\mathcal{H} \times L^{2}\left(0,1 ;\left(L^{2}(\Gamma)\right)^{3}\right)
$$

endowed with the scalar product

$$
\begin{aligned}
\left\langle(\mathbf{E}, \mathbf{H}, \mathbf{Z})^{T},(\tilde{\mathbf{E}}, \tilde{\mathbf{H}}, \tilde{\mathbf{Z}})^{T}\right\rangle_{\mathscr{H}} & :=\left\langle(\mathbf{E}, \mathbf{H})^{T},(\tilde{\mathbf{E}}, \tilde{\mathbf{H}})^{T}\right\rangle_{\mathcal{H}} \\
& +\tau \int_{0}^{1} \int_{\Gamma}(\mathbf{E}(t-\tau s) \times \boldsymbol{\nu}) \cdot(\tilde{\mathbf{E}}(t-\tau s) \times \boldsymbol{\nu}) \mathrm{d} \mathbf{x d} s .
\end{aligned}
$$

Letting formally

$$
\mathbf{V}(t, \cdot):=\left(\begin{array}{c}
\mathbf{E}(t, \cdot) \\
\mathbf{H}(t, \cdot) \\
(0,1) \ni s \mapsto \mathbf{Z}(t, s, \cdot)
\end{array}\right) \equiv\left(\begin{array}{c}
\mathbf{E}(t, \cdot) \\
\mathbf{H}(t, \cdot) \\
\left.\left.(0,1) \ni s \mapsto(\mathbf{E}(t-\tau s) \times \boldsymbol{\nu})\right|_{\Gamma}\right)
\end{array}\right)
$$


we define the operator

$$
\mathscr{A}: D(\mathscr{A}) \subset \mathscr{H} \rightarrow \mathscr{H}, \quad(\mathbf{E}, \mathbf{H}, \mathbf{Z})^{T} \mapsto\left(\begin{array}{c}
\mathcal{A}(\mathbf{E}, \mathbf{H})^{T} \\
\frac{1}{\tau} \partial_{s} \mathbf{Z}
\end{array}\right)
$$

with the domain

$$
\begin{aligned}
D(\mathscr{A}):=\left\{(\mathbf{E}, \mathbf{H}, \mathbf{Z})^{T} \in \mathscr{H} \mid\right. & \left(\mathcal{A}(\mathbf{E}, \mathbf{H})^{T}, \frac{1}{\tau} \partial_{s} \mathbf{Z}\right)^{T} \in \mathscr{H}, \quad \mathbf{E} \times\left.\boldsymbol{\nu}\right|_{\Gamma}, \mathbf{H} \times\left.\boldsymbol{\nu}\right|_{\Gamma} \in\left(L^{2}(\Gamma)\right)^{3}, \\
& \mathbf{H} \times \boldsymbol{\nu}+\gamma_{1} \mathbf{g}(\mathbf{E} \times \boldsymbol{\nu}) \times \boldsymbol{\nu}+\gamma_{2} \mathbf{g}\left(\left.\mathbf{Z}\right|_{s=1}\right) \times \boldsymbol{\nu}=\mathbf{0} \text { on } \Gamma, \\
& \left.\left.\mathbf{Z}\right|_{s=0}=\mathbf{E} \times \boldsymbol{\nu}\right\}
\end{aligned}
$$

The latter explicitly reads as

$$
\begin{aligned}
D(\mathscr{A}):=\left\{(\mathbf{E}, \mathbf{H}, \mathbf{Z})^{T} \in \mathscr{H} \mid\right. & \mathbf{E}, \mathbf{H} \in H(\operatorname{curl}, G), \quad \mathbf{Z} \in H^{1}\left(0,1 ;\left(L^{2}(\Gamma)\right)^{3}\right), \\
& \mathbf{E} \times\left.\boldsymbol{\nu}\right|_{\Gamma}, \mathbf{H} \times\left.\boldsymbol{\nu}\right|_{\Gamma} \in\left(L^{2}(\Gamma)\right)^{3}, \\
& \mathbf{H} \times \boldsymbol{\nu}+\gamma_{1} \mathbf{g}(\mathbf{E} \times \boldsymbol{\nu}) \times \boldsymbol{\nu}+\gamma_{2} \mathbf{g}\left(\left.\mathbf{Z}\right|_{s=1}\right) \times \boldsymbol{\nu}=\mathbf{0} \text { on } \Gamma, \\
& \left.\left.\mathbf{Z}\right|_{s=0}=\mathbf{E} \times \boldsymbol{\nu}\right\} .
\end{aligned}
$$

Equations (1.9)- 1.13 can equivalently be written as an abstract evolution equation

$$
\partial_{t} \mathbf{V}(t)+\mathscr{A}(\mathbf{V}(t))=\mathbf{0} \text { for } t>0, \quad \mathbf{V}(0)=\mathbf{V}^{0}
$$

with $\mathbf{V}^{0}:=\left(\mathbf{E}^{0}, \mathbf{H}^{0}, \boldsymbol{\Phi}^{0}\right)^{T}$.

Assumption 2.1 (Tensor fields $\varepsilon$ and $\boldsymbol{\mu})$. Let $\boldsymbol{\varepsilon}, \boldsymbol{\mu} \in C^{0}\left(\bar{G}, \mathbb{R}^{3 \times 3}\right)$ satisfy

$$
(\varepsilon(\mathbf{x}))^{T}=\varepsilon(\mathbf{x}) \text { and }(\boldsymbol{\mu}(\mathbf{x}))^{T}=\boldsymbol{\mu}(\mathbf{x}) \text { for } \mathbf{x} \in \bar{G}
$$

as well as

$$
\lambda_{\min }(\varepsilon)>0 \text { and } \lambda_{\min }(\boldsymbol{\mu})>0,
$$

where

$$
\lambda_{\min }(\boldsymbol{\varphi}):=\min _{x \in \bar{G}} \min _{|\boldsymbol{\xi}|=1} \boldsymbol{\xi} \cdot(\boldsymbol{\varphi}(\mathbf{x}) \boldsymbol{\xi}) \text { for } \boldsymbol{\varphi} \in C^{0}\left(\bar{G}, \mathbb{R}^{3 \times 3}\right)
$$

Denote

$$
\alpha=\min \left\{\lambda_{\min }(\varepsilon), \lambda_{\min }(\boldsymbol{\mu})\right\} .
$$

Assumption 2.2 (Nonlinearity $\mathbf{g}(\cdot))$. Suppose the nonlinear function $\mathbf{g}: \mathbb{R}^{3} \rightarrow \mathbb{R}^{3}$ satisfies:

1. $\mathbf{g}(\mathbf{0})=\mathbf{0}$,

2. There exists $c_{1}>0$ such that $(\mathbf{g}(\mathbf{E})-\mathbf{g}(\tilde{\mathbf{E}})) \cdot(\mathbf{E}-\tilde{\mathbf{E}}) \geq c_{1}|\mathbf{E}-\tilde{\mathbf{E}}|^{2}$ for any $\mathbf{E}, \tilde{\mathbf{E}} \in \mathbb{R}^{3}$,

3. There exists $c_{2}>0$ such that $|\mathbf{g}(\mathbf{E})-\mathbf{g}(\tilde{\mathbf{E}})| \leq c_{2}|\mathbf{E}-\tilde{\mathbf{E}}|$ for any $\mathbf{E}, \tilde{\mathbf{E}} \in \mathbb{R}^{3}$.

Remark 2.3. In contrast to the wave equation, which is known [17] to admit feedback functions with a superlinear growth rate (in $y$, not $y_{t}$ ), this is no longer true for Maxwell's equations since superlinear terms can cause the solution to leave the basic $L^{2}$-space thus destroying the well-posedness. In this sense, the results of our paper appear to be optimal - at least at the basic energy level. 
The following two lemmas are quoted from [9].

Lemma 2.4. For all $\mathbf{E}, \mathbf{H} \in H(\operatorname{curl}, G)$ with $\mathbf{E} \times\left.\boldsymbol{\nu}\right|_{\Gamma}, \mathbf{H} \times\left.\boldsymbol{\nu}\right|_{\Gamma} \in\left(L^{2}(\Gamma)\right)^{3}$, we have

$$
\int_{G}(\operatorname{curl} \mathbf{E} \cdot \mathbf{H}-\operatorname{curl} \mathbf{H} \cdot \mathbf{E}) \mathrm{d} \mathbf{x}=\int_{\Gamma}(\mathbf{H} \times \boldsymbol{\nu}) \cdot \mathbf{E} \mathrm{d} \mathbf{x} .
$$

Let $P_{\boldsymbol{\varepsilon}}$ denote the orthogonal projection on $H\left(\operatorname{div}_{\boldsymbol{\varepsilon}} 0, G\right)$ in $\left(L^{2}(G)\right)^{3}$.

Lemma 2.5. The image $P_{\boldsymbol{\varepsilon}}(\mathcal{D}(G))^{3}$ is dense in $H\left(\operatorname{div}_{\boldsymbol{\varepsilon}} 0, G\right)$. The domain of the operator $\mathscr{A}$ is dense in $\mathscr{H}$.

Remark 2.6. For all $\chi \in C^{\infty}(\bar{G})$, we have $\operatorname{curl}\left(P_{\varepsilon} \chi\right)=\operatorname{curl} \chi$ in $G$ and $\left(P_{\varepsilon} \chi\right) \times \boldsymbol{\nu}=\chi \times \boldsymbol{\nu}$ on $\Gamma$.

Now, we can prove the following lemma.

Lemma 2.7. There exists a positive number $C$ such that $C \cdot i d+\mathscr{A}$ is a maximal monotone operator.

Proof. Monotonicity: Consider a new inner product on $\mathscr{H}$ defined via

$$
\begin{aligned}
\left\langle(\mathbf{E}, \mathbf{H}, \mathbf{Z})^{T},(\tilde{\mathbf{E}}, \tilde{\mathbf{H}}, \tilde{\mathbf{Z}})^{T}\right\rangle_{\tilde{\mathscr{H}}} & :=\left\langle(\mathbf{E}, \mathbf{H})^{T},(\tilde{\mathbf{E}}, \tilde{\mathbf{H}})^{T}\right\rangle_{\mathcal{H}} \\
& +\xi \tau \int_{0}^{1} \int_{\Gamma} e^{c s}(\mathbf{E}(t-\tau s) \times \boldsymbol{\nu}) \cdot(\tilde{\mathbf{E}}(t-\tau s) \times \boldsymbol{\nu}) \mathrm{d} \mathbf{x d} s .
\end{aligned}
$$

Here $c, \xi$ are positive numbers and will be chosen later. Obviously, $\langle\cdot, \cdot\rangle_{\tilde{\mathscr{H}}}$ is equivalent with the original inner product $\langle\cdot, \cdot\rangle_{\mathscr{H}}$.

First, we show that $C \cdot i d+\mathscr{A}$ is a monotone operator for some $C>0$. For all $(\mathbf{E}, \mathbf{H}, \mathbf{Z})^{T}$, $\left(\mathbf{E}^{\prime}, \mathbf{H}^{\prime}, \mathbf{Z}^{\prime}\right)^{T} \in D(\mathscr{A})$, letting $(\tilde{\mathbf{E}}, \tilde{\mathbf{H}}, \tilde{\mathbf{Z}})^{T}=(\mathbf{E}, \mathbf{H}, \mathbf{Z})^{T}-\left(\mathbf{E}^{\prime}, \mathbf{H}^{\prime}, \mathbf{Z}^{\prime}\right)^{T}$, we obtain

$$
\begin{aligned}
& \left\langle(C \cdot i d+\mathscr{A})\left(\begin{array}{l}
\mathbf{E} \\
\mathbf{H} \\
\mathbf{Z}
\end{array}\right)-(C \cdot i d+\mathscr{A})\left(\begin{array}{l}
\mathbf{E}^{\prime} \\
\mathbf{H}^{\prime} \\
\mathbf{Z}^{\prime}
\end{array}\right),\left(\begin{array}{l}
\mathbf{E} \\
\mathbf{H} \\
\mathbf{Z}
\end{array}\right)-\left(\begin{array}{l}
\mathbf{E}^{\prime} \\
\mathbf{H}^{\prime} \\
\mathbf{Z}^{\prime}
\end{array}\right)\right\rangle_{\tilde{\mathscr{H}}} \\
& =C\left\|\left(\begin{array}{c}
\tilde{\mathbf{E}} \\
\tilde{\mathbf{H}} \\
\tilde{\mathbf{Z}}
\end{array}\right)\right\|_{\tilde{\mathscr{H}}}^{2}+\left\langle\mathscr{A}\left(\begin{array}{c}
\tilde{\mathbf{E}} \\
\tilde{\mathbf{H}} \\
\tilde{\mathbf{Z}}
\end{array}\right),\left(\begin{array}{c}
\tilde{\mathbf{E}} \\
\tilde{\mathbf{H}} \\
\tilde{\mathbf{Z}}
\end{array}\right)\right\rangle_{\tilde{\mathscr{H}}} \\
& =C\left\|\left(\begin{array}{c}
\tilde{\mathbf{E}} \\
\tilde{\mathbf{H}} \\
\tilde{\mathbf{Z}}
\end{array}\right)\right\|_{\tilde{\mathscr{H}}}^{2}+\left\langle\left(\begin{array}{c}
-\varepsilon^{-1} \operatorname{curl} \tilde{\mathbf{H}} \\
\boldsymbol{\mu}^{-1} \operatorname{curl} \tilde{\mathbf{E}} \\
\tau^{-1} \partial_{s} \tilde{\mathbf{Z}}
\end{array}\right),\left(\begin{array}{c}
\tilde{\mathbf{E}} \\
\tilde{\mathbf{H}} \\
\tilde{\mathbf{Z}}
\end{array}\right)\right\rangle_{\tilde{\mathscr{H}}} \\
& =C\left\|\left(\begin{array}{c}
\tilde{\mathbf{E}} \\
\tilde{\mathbf{H}} \\
\tilde{\mathbf{Z}}
\end{array}\right)\right\|_{\tilde{\mathscr{H}}}^{2}+\int_{G}(\operatorname{curl} \tilde{\mathbf{E}} \cdot \tilde{\mathbf{H}}-\operatorname{curl} \tilde{\mathbf{H}} \cdot \tilde{\mathbf{E}}) \mathrm{d} \mathbf{x}+\xi \int_{0}^{1} \int_{\Gamma} e^{c s} \partial_{s} \tilde{\mathbf{Z}} \cdot \tilde{\mathbf{Z}} \mathrm{d} \mathbf{x d} s .
\end{aligned}
$$

Using Lemma 2.4 and the boundary condition from Equation 1.11, we get

$$
\begin{aligned}
& \int_{G}(\operatorname{curl} \tilde{\mathbf{E}} \cdot \tilde{\mathbf{H}}-\operatorname{curl} \tilde{\mathbf{H}} \cdot \tilde{\mathbf{E}}) \mathbf{d} \mathbf{x}=\int_{\Gamma} \tilde{\mathbf{H}} \times \boldsymbol{\nu} \cdot \tilde{\mathbf{E}} \mathrm{d} \mathbf{x} \\
& =\int_{\Gamma}\left(\gamma_{1} \mathbf{g}\left(\mathbf{E}^{\prime} \times \boldsymbol{\nu}\right) \times \boldsymbol{\nu}+\gamma_{2} \mathbf{g}\left(\left.\mathbf{Z}^{\prime}\right|_{s=1}\right) \times \boldsymbol{\nu}-\gamma_{1} \mathbf{g}(\mathbf{E} \times \boldsymbol{\nu}) \times \boldsymbol{\nu}-\gamma_{2} \mathbf{g}\left(\left.\mathbf{Z}\right|_{s=1}\right) \times \boldsymbol{\nu}\right) \cdot\left(\mathbf{E}-\mathbf{E}^{\prime}\right) \mathrm{d} \mathbf{x} \\
& =\int_{\Gamma}\left(\gamma_{1} \mathbf{g}\left(\mathbf{E}^{\prime} \times \boldsymbol{\nu}\right)+\gamma_{2} \mathbf{g}\left(\left.\mathbf{Z}^{\prime}\right|_{s=1}\right)-\gamma_{1} \mathbf{g}(\mathbf{E} \times \boldsymbol{\nu})-\gamma_{2} \mathbf{g}\left(\left.\mathbf{Z}\right|_{s=1}\right)\right) \times \boldsymbol{\nu} \cdot\left(\mathbf{E}-\mathbf{E}^{\prime}\right) \mathrm{d} \mathbf{x}
\end{aligned}
$$




$$
\begin{aligned}
& =\int_{\Gamma}\left(\gamma_{1} \mathbf{g}(\mathbf{E} \times \boldsymbol{\nu})+\gamma_{2} \mathbf{g}\left(\left.\mathbf{Z}\right|_{s=1}\right)-\gamma_{1} \mathbf{g}\left(\mathbf{E}^{\prime} \times \boldsymbol{\nu}\right)-\gamma_{2} \mathbf{g}\left(\left.\mathbf{Z}^{\prime}\right|_{s=1}\right)\right) \cdot\left(\mathbf{E}-\mathbf{E}^{\prime}\right) \times \boldsymbol{\nu} \mathrm{d} \mathbf{x} \\
& =\int_{\Gamma} \gamma_{1}\left(\mathbf{g}(\mathbf{E} \times \boldsymbol{\nu})-\mathbf{g}\left(\mathbf{E}^{\prime} \times \boldsymbol{\nu}\right)\right) \cdot\left(\mathbf{E} \times \boldsymbol{\nu}-\mathbf{E}^{\prime} \times \boldsymbol{\nu}\right)+\gamma_{2}\left(\mathbf{g}\left(\left.\mathbf{Z}\right|_{s=1}\right)-\mathbf{g}\left(\left.\mathbf{Z}^{\prime}\right|_{s=1}\right)\right) \cdot\left(\left.\tilde{\mathbf{Z}}\right|_{s=0}\right) \mathrm{d} \mathbf{x} .
\end{aligned}
$$

Recalling Assumption 2.2 and using Cauchy \& Schwarz' inequality, the latter integral can be estimated both on the low

$$
\begin{aligned}
\int_{\Gamma} \gamma_{1}\left(\mathbf{g}(\mathbf{E} \times \boldsymbol{\nu})-\mathbf{g}\left(\mathbf{E}^{\prime} \times \boldsymbol{\nu}\right)\right) \cdot(\mathbf{E} \times \boldsymbol{\nu} & \left.-\mathbf{E}^{\prime} \times \boldsymbol{\nu}\right) \mathrm{d} \mathbf{x} \geq \int_{\Gamma} \gamma_{1} c_{1}\left|\mathbf{E} \times \boldsymbol{\nu}-\mathbf{E}^{\prime} \times \boldsymbol{\nu}\right|^{2} \mathrm{~d} \mathbf{x} \\
& =\left.\gamma_{1} c_{1} \int_{\Gamma} \tilde{\mathbf{Z}}^{2}\right|_{s=0} \mathrm{~d} \mathbf{x}=\gamma_{1} c_{1}\left\|\left.\tilde{\mathbf{Z}}\right|_{s=0}\right\|_{\left(L^{2}(\Gamma)\right)^{3}}^{2}
\end{aligned}
$$

and the high side

$$
\begin{aligned}
\int_{\Gamma} \gamma_{2}\left(\mathbf{g}\left(\left.\mathbf{Z}\right|_{s=1}\right)\right. & \left.-\mathbf{g}\left(\left.\mathbf{Z}^{\prime}\right|_{s=1}\right)\right) \cdot\left(\left.\tilde{\mathbf{Z}}\right|_{s=0}\right) \mathrm{d} \mathbf{x} \\
& \geq-\gamma_{2}\left(\left.\int_{\Gamma}\left(\mathbf{g}\left(\left.\mathbf{Z}\right|_{s=1}\right)-\mathbf{g}\left(\left.\mathbf{Z}^{\prime}\right|_{s=1}\right)\right)^{2} \mathrm{~d} \mathbf{x} \int_{\Gamma} \tilde{\mathbf{Z}}^{2}\right|_{s=0} \mathrm{~d} \mathbf{x}\right)^{\frac{1}{2}} \\
& \geq-\gamma_{2}\left(\left.\int_{\Gamma}\left(c_{2}\left(\left.\mathbf{Z}\right|_{s=1}-\left.\mathbf{Z}^{\prime}\right|_{s=1}\right)\right)^{2} \mathrm{~d} \mathbf{x} \int_{\Gamma} \tilde{\mathbf{Z}}^{2}\right|_{s=0} \mathrm{~d} \mathbf{x}\right)^{\frac{1}{2}} \\
& =-\gamma_{2} c_{2}\left\|\left.\tilde{\mathbf{Z}}\right|_{s=1}\right\|_{\left(L^{2}(\Gamma)\right)^{3}} \cdot\left\|\left.\tilde{\mathbf{Z}}\right|_{s=0}\right\|_{\left(L^{2}(\Gamma)\right)^{3}} .
\end{aligned}
$$

Now, consider the latter term in Equation (2.4). Integrating by parts, we get

$$
\begin{aligned}
\xi \int_{0}^{1} \int_{\Gamma} e^{c s} \partial_{s} \tilde{\mathbf{Z}} \cdot \tilde{\mathbf{Z}} \mathrm{d} \mathbf{x} \mathrm{d} s & =\frac{\xi}{2} \int_{\Gamma} \int_{0}^{1} e^{c s} \partial_{s}\left(\tilde{\mathbf{Z}}^{2}\right) \mathrm{d} s \mathrm{~d} \mathbf{x} \\
& =\frac{\xi}{2} \int_{\Gamma}\left(\left.e^{c s} \tilde{\mathbf{Z}}^{2}\right|_{s=0} ^{s=1}-\int_{0}^{1} c e^{c s} \tilde{\mathbf{Z}}^{2} \mathrm{~d} s\right) \mathrm{d} \mathbf{x} \\
& =\frac{\xi}{2} \int_{\Gamma}\left(\left.e^{c} \tilde{\mathbf{Z}}^{2}\right|_{s=1}-\left.\tilde{\mathbf{Z}}^{2}\right|_{s=0}-\int_{0}^{1} c e^{c s} \tilde{\mathbf{Z}}^{2} \mathrm{~d} s\right) \mathrm{d} \mathbf{x} \\
& =\frac{e^{c} \xi}{2}\left\|\left.\tilde{\mathbf{Z}}\right|_{s=1}\right\|_{\left(L^{2}(\Gamma)\right)^{3}}^{2}-\frac{\xi}{2}\left\|\left.\tilde{\mathbf{Z}}\right|_{s=0}\right\|_{\left(L^{2}(\Gamma)\right)^{3}}^{2}-\frac{\xi c}{2} \int_{\Gamma} \int_{0}^{1} e^{c s} \tilde{\mathbf{Z}}^{2} \mathrm{~d} s \mathrm{~d} \mathbf{x} .
\end{aligned}
$$

Recalling Equations 2.5-2.8, we obtain

$$
\begin{aligned}
& C\left\|\left(\begin{array}{c}
\tilde{\mathbf{E}} \\
\tilde{\mathbf{H}} \\
\tilde{\mathbf{Z}}
\end{array}\right)\right\|_{\tilde{\mathscr{H}}}^{2}+\int_{G}(\operatorname{curl} \tilde{\mathbf{E}} \cdot \tilde{\mathbf{H}}-\operatorname{curl} \tilde{\mathbf{H}} \cdot \tilde{\mathbf{E}}) \mathrm{d} \mathbf{x}+\xi \int_{0}^{1} \int_{\Gamma} e^{c s} \partial_{s} \tilde{\mathbf{Z}} \cdot \tilde{\mathbf{Z}} \mathrm{d} \mathbf{x} \mathrm{d} s \\
& \geq C\left\|\left(\begin{array}{c}
\tilde{\mathbf{E}} \\
\tilde{\mathbf{H}}
\end{array}\right)\right\|_{\mathcal{H}}^{2}+C \xi \tau \int_{0}^{1} \int_{\Gamma} e^{c s} \tilde{\mathbf{Z}}^{2} \mathrm{~d} \mathbf{x} \mathrm{d} s+\left(\gamma_{1} c_{1}-\frac{\xi}{2}\right)\left\|\left.\tilde{\mathbf{Z}}\right|_{s=0}\right\|_{\left(L^{2}(\Gamma)\right)^{3}}^{2}+\frac{\xi e^{c}}{2}\left\|\left.\tilde{\mathbf{Z}}\right|_{s=1}\right\|_{\left(L^{2}(\Gamma)\right)^{3}}^{2} \\
& -\frac{c \xi}{2} \int_{0}^{1} \int_{\Gamma} e^{c s} \tilde{\mathbf{Z}}^{2} \mathrm{~d} \mathbf{x} \mathrm{d} s-\gamma_{2} c_{2}\left\|\left.\tilde{\mathbf{Z}}\right|_{s=1}\right\|_{\left(L^{2}(\Gamma)\right)^{3}} \cdot\left\|\left.\tilde{\mathbf{Z}}\right|_{s=0}\right\|_{\left(L^{2}(\Gamma)\right)^{3}} .
\end{aligned}
$$

Taking now $\xi<2 \gamma_{1} c_{1}$ and applying Cauchy \& Schwarz' inequality, we arrive at

$$
C\left\|\left(\begin{array}{c}
\tilde{\mathbf{E}} \\
\tilde{\mathbf{H}}
\end{array}\right)\right\|_{\mathcal{H}}^{2}+C \xi \tau \int_{0}^{1} \int_{\Gamma} e^{c s} \tilde{\mathbf{Z}}^{2} \mathrm{~d} \mathbf{x} \mathrm{d} s+\left(\gamma_{1} c_{1}-\frac{\xi}{2}\right)\left\|\left.\tilde{\mathbf{Z}}\right|_{s=0}\right\|_{\left(L^{2}(\Gamma)\right)^{3}}^{2}+\frac{\xi e^{c}}{2}\left\|\left.\tilde{\mathbf{Z}}\right|_{s=1}\right\|_{\left(L^{2}(\Gamma)\right)^{3}}^{2}
$$




$$
\begin{aligned}
& -\frac{c \xi}{2} \int_{0}^{1} \int_{\Gamma} e^{c s} \tilde{\mathbf{Z}}^{2} \mathrm{~d} \mathbf{x} \mathrm{d} s-\gamma_{2} c_{2}\left\|\left.\tilde{\mathbf{Z}}\right|_{s=1}\right\|_{\left(L^{2}(\Gamma)\right)^{3}} \cdot\left\|\left.\tilde{\mathbf{Z}}\right|_{s=0}\right\|_{\left(L^{2}(\Gamma)\right)^{3}} \\
& \geq C\left\|\left(\begin{array}{c}
\tilde{\mathbf{E}} \\
\tilde{\mathbf{H}}
\end{array}\right)\right\|_{\mathcal{H}}^{2}+2\left(\left(\gamma_{1} c_{1}-\frac{\xi}{2}\right) \frac{\xi e^{c}}{2}\right)^{\frac{1}{2}}\left\|\left.\tilde{\mathbf{Z}}\right|_{s=1}\right\|_{\left(L^{2}(\Gamma)\right)^{3}} \cdot\left\|\left.\tilde{\mathbf{Z}}\right|_{s=0}\right\|_{\left(L^{2}(\Gamma)\right)^{3}} \\
& +\xi\left(C \tau-\frac{c}{2}\right) \int_{0}^{1} \int_{\Gamma} e^{c s} \tilde{\mathbf{Z}}^{2} \mathrm{~d} \mathbf{x} \mathrm{d} s-\gamma_{2} c_{2}\left\|\left.\tilde{\mathbf{Z}}\right|_{s=1}\right\|_{\left(L^{2}(\Gamma)\right)^{3}} \cdot\left\|\left.\tilde{\mathbf{Z}}\right|_{s=0}\right\|_{\left(L^{2}(\Gamma)\right)^{3}}
\end{aligned}
$$

Finally, selecting $c$ such that $2\left(\left(\gamma_{1} c_{1}-\frac{\xi}{2}\right) \frac{\xi e^{c}}{2}\right)^{\frac{1}{2}} \geq \gamma_{2} c_{2}$ and then choosing $C>\frac{c}{2 \tau}$, the right hand side of Equation (2.9) is rendered positive implying the monotonicity of $\mathscr{A}$.

Maximality: By virtue of Browder \& Minty's Theorem [1, Theorem 2.2], it suffices to prove $(C+\lambda) \cdot i d+\mathscr{A}$ is surjective for at least one $\lambda>0$, i.e., for any $\left(\mathbf{F}_{1}, \mathbf{F}_{2}, \mathbf{F}_{3}\right)^{T} \in \mathscr{H}$, we need to find $(\mathbf{E}, \mathbf{H}, \mathbf{Z}) \in D(\mathscr{A})$ such that

$$
((C+1) \cdot i d+\mathscr{A})\left(\begin{array}{l}
\mathbf{E} \\
\mathbf{H} \\
\mathbf{Z}
\end{array}\right)=\left(\begin{array}{l}
\mathbf{F}_{1} \\
\mathbf{F}_{2} \\
\mathbf{F}_{3}
\end{array}\right) .
$$

Let $b=C+1$. From Equation 2.10 , we have $b \mathbf{Z}+\tau^{-1} \partial_{s} \mathbf{Z}=\mathbf{F}_{3}$, whence we easily get

$$
\mathbf{Z}(t, s, \mathbf{x})=e^{-\tau b s}\left(\int_{0}^{s} \mathbf{F}_{3}(t, s, \mathbf{x}) e^{\tau b r} \mathrm{~d} r+\mathbf{E}(t, \mathbf{x}) \times \boldsymbol{\nu}\right) .
$$

In particular,

$$
\begin{aligned}
& \left.\mathbf{Z}(t, s, \mathbf{x})\right|_{s=1}=e^{-\tau b}\left(\int_{0}^{1} \mathbf{F}_{3}(t, r, \mathbf{x}) e^{\tau b r} \mathrm{~d} r+\mathbf{E}(t, \mathbf{x}) \times \boldsymbol{\nu}\right), \\
& \left.\mathbf{Z}(t, s, \mathbf{x})\right|_{s=0}=\mathbf{E}(t, \mathbf{x}) \times \boldsymbol{\nu} .
\end{aligned}
$$

Further, using Equation 2.10, we obtain

$$
\mathbf{H}=b^{-1}\left(\mathbf{F}_{2}-\boldsymbol{\mu}^{-1} \operatorname{curl} \mathbf{E}\right)
$$

to arrive at

$$
b^{2} \boldsymbol{\varepsilon} \mathbf{E}-\operatorname{curl}\left(\mathbf{F}_{2}-\boldsymbol{\mu}^{-1} \operatorname{curl} \mathbf{E}\right)=b \varepsilon \mathbf{F}_{1} .
$$

The latter equation is formally equivalent with

$$
b^{2} \varepsilon \mathbf{E}+\operatorname{curl}\left(\boldsymbol{\mu}^{-1} \operatorname{curl} \mathbf{E}\right)=b \boldsymbol{\varepsilon} \mathbf{F}_{1}+\operatorname{curl} \mathbf{F}_{2},
$$

while the boundary condition in Equation (1.11) can formally be transformed to

$$
-b^{-1} \boldsymbol{\mu}^{-1} \operatorname{curl} \mathbf{E} \times \boldsymbol{\nu}+\gamma_{1} \mathbf{g}\left(\left.\mathbf{Z}\right|_{s=0}\right) \times \boldsymbol{\nu}+\gamma_{2} \mathbf{g}\left(\left.\mathbf{Z}\right|_{s=1}\right) \times \boldsymbol{\nu}=-b^{-1} \mathbf{F}_{2} \times \boldsymbol{\nu},
$$

where $\left.\mathbf{Z}\right|_{s=1}$ and $\left.\mathbf{Z}\right|_{s=0}$ are given by Equations (2.12) and 2.13), respectively.

Define the Hilbert space

$$
W_{\boldsymbol{\varepsilon}}=\left\{\mathbf{E} \in\left(L^{2}(G)\right)^{3} \mid \operatorname{curl} \mathbf{E} \in\left(L^{2}(G)\right)^{3}, \operatorname{div}(\varepsilon \mathbf{E}) \in L^{2}(G), \mathbf{E} \times \boldsymbol{\nu} \in\left(L^{2}(\Gamma)\right)^{3}\right\}
$$

endowed with the norm

$$
\|\mathbf{E}\|_{W_{\boldsymbol{\varepsilon}}}^{2}=\int_{G}|\mathbf{E}|^{2}+|\operatorname{curl} \mathbf{E}|^{2}+|\operatorname{div}(\varepsilon \mathbf{E})|^{2} \mathrm{~d} \mathbf{x}+\int_{\Gamma}|\mathbf{E} \times \boldsymbol{\nu}|^{2} \mathrm{~d} \mathbf{x} .
$$


Consider the variational problem: Find $\mathbf{E} \in W_{\varepsilon}$ such that

$$
\mathfrak{a}\left(\mathbf{E}, \mathbf{E}^{\prime}\right)=\int_{G} b \varepsilon \mathbf{F}_{1} \cdot \mathbf{E}^{\prime}+\mathbf{F}_{2} \cdot \operatorname{curl} \mathbf{E}^{\prime} \mathrm{d} \mathbf{x} \text { for any } \mathbf{E}^{\prime} \in W_{\varepsilon} .
$$

Here, the nonlinear form $\mathfrak{a}(\cdot, \cdot)$ is defined by

$$
\begin{aligned}
\mathfrak{a}\left(\mathbf{E}, \mathbf{E}^{\prime}\right):= & \int_{G} b^{2} \varepsilon \mathbf{E} \cdot \mathbf{E}^{\prime}+\boldsymbol{\mu}^{-1} \operatorname{curl} \mathbf{E} \cdot \operatorname{curl} \mathbf{E}^{\prime}+s \operatorname{div}(\varepsilon \mathbf{E}) \operatorname{div}\left(\varepsilon \mathbf{E}^{\prime}\right) \mathrm{d} \mathbf{x} \\
& +b \int_{\Gamma}\left(\mathbf{E}^{\prime} \times \boldsymbol{\nu}\right) \cdot\left(\gamma_{1} \mathbf{g}\left(\left.\mathbf{Z}\right|_{s=0}\right)+\gamma_{2} \mathbf{g}\left(\left.\mathbf{Z}\right|_{s=1}\right)\right) \mathrm{d} \mathbf{x}
\end{aligned}
$$

where $\left.\mathbf{Z}\right|_{s=1}$ and $\left.\mathbf{Z}\right|_{s=0}$ are given by Equations (2.12) and (2.13), respectively, and $s$ is a positive number to be chosen later.

Similar to [9], consider the operator

$$
\mathcal{B}: W_{\varepsilon} \rightarrow W_{\varepsilon}^{\prime}, \quad \mathcal{B} u(v)=\mathfrak{a}(u, v)
$$

Observing that right-hand side of Equation 2.20 belongs to the space $W_{\varepsilon}^{\prime}$, the solvability of Equation 2.20 needs to follow from surjectivity of the operator $\mathcal{B}$. Using [24, Corollary 2.2] and the fact that strong monotonicity implies coercivity, it is sufficient to prove $\mathcal{B}$ is strongly monotone, hemicontinuous and bounded.

Strong monotonicity: For any $\mathbf{E}, \mathbf{E}^{\prime} \in W_{\varepsilon}$, letting $\tilde{\mathbf{E}}=\mathbf{E}-\mathbf{E}^{\prime}$, we have

$$
\begin{aligned}
\left\langle\mathcal{B} \mathbf{E}-\mathcal{B} \mathbf{E}^{\prime}, \mathbf{E}\right. & \left.-\mathbf{E}^{\prime}\right\rangle_{W_{\varepsilon}^{\prime} \times W_{\varepsilon}}=\left\langle\mathcal{B} \mathbf{E}, \mathbf{E}-\mathbf{E}^{\prime}\right\rangle_{W_{\varepsilon}^{\prime} \times W_{\varepsilon}}-\left\langle\mathcal{B} \mathbf{E}^{\prime}, \mathbf{E}-\mathbf{E}^{\prime}\right\rangle_{W_{\boldsymbol{\varepsilon}}^{\prime} \times W_{\varepsilon}} \\
& =\mathfrak{a}\left(\mathbf{E}, \mathbf{E}-\mathbf{E}^{\prime}\right)-\mathfrak{a}\left(\mathbf{E}^{\prime}, \mathbf{E}-\mathbf{E}^{\prime}\right) \\
& =\int_{G} b^{2} \varepsilon \mathbf{E} \cdot \tilde{\mathbf{E}}+\boldsymbol{\mu}^{-1} \operatorname{curl} \mathbf{E} \cdot \operatorname{curl} \tilde{\mathbf{E}}+s \operatorname{div}(\varepsilon \mathbf{E}) \operatorname{div}(\varepsilon \tilde{\mathbf{E}}) \mathrm{d} \mathbf{x}+ \\
& +b \int_{\Gamma}(\tilde{\mathbf{E}} \times \boldsymbol{\nu}) \cdot\left(\gamma_{1} \mathbf{g}(\mathbf{E} \times \boldsymbol{\nu})+\gamma_{2} \mathbf{g}\left(e^{-\tau b}\left(\int_{0}^{1} \mathbf{F}_{3}(r) e^{\tau b r} \mathrm{~d} r+\mathbf{E} \times \boldsymbol{\nu}\right)\right)\right) \mathrm{d} \mathbf{x} \\
& -\int_{G} b^{2} \varepsilon \mathbf{E}^{\prime} \cdot \tilde{\mathbf{E}}+\boldsymbol{\mu}^{-1} \operatorname{curl} \mathbf{E}^{\prime} \cdot \operatorname{curl} \tilde{\mathbf{E}}+s \operatorname{div}\left(\varepsilon \mathbf{E}^{\prime}\right) \operatorname{div}(\varepsilon \tilde{\mathbf{E}}) \mathrm{d} \mathbf{x}+ \\
& -b \int_{\Gamma}(\tilde{\mathbf{E}} \times \boldsymbol{\nu}) \cdot\left(\gamma_{1} \mathbf{g}\left(\mathbf{E}^{\prime} \times \boldsymbol{\nu}\right)+\gamma_{2} \mathbf{g}\left(e^{-\tau b}\left(\int_{0}^{1} F_{3}(r) e^{\tau b r} \mathrm{~d} r+\mathbf{E}^{\prime} \times \boldsymbol{\nu}\right)\right)\right) \mathrm{d} \mathbf{x} \\
& =\int_{G} b^{2} \varepsilon \tilde{\mathbf{E}} \cdot \tilde{\mathbf{E}}+\boldsymbol{\mu}^{-1} \operatorname{curl} \tilde{\mathbf{E}} \cdot \operatorname{curl} \tilde{\mathbf{E}}+s \operatorname{div}\left(\varepsilon \tilde{\mathbf{E}}^{2}\right) \operatorname{div}(\varepsilon \tilde{\mathbf{E}}) \mathrm{d} \mathbf{x}+ \\
& +b \int_{\Gamma}(\tilde{\mathbf{E}} \times \boldsymbol{\nu}) \cdot\left(\gamma_{1} \mathbf{g}(\mathbf{E} \times \boldsymbol{\nu})+\gamma_{2} \mathbf{g}\left(e^{-\tau b}\left(\int_{0}^{1} \mathbf{F}_{3}(r) e^{\tau b r} \mathrm{~d} r+\mathbf{E} \times \boldsymbol{\nu}\right)\right)\right) \mathrm{d} \mathbf{x} \\
& -b \int_{\Gamma}(\tilde{\mathbf{E}} \times \boldsymbol{\nu}) \cdot\left(\gamma_{1} \mathbf{g}\left(\mathbf{E}^{\prime} \times \boldsymbol{\nu}\right)+\gamma_{2} \mathbf{g}\left(e^{-\tau b}\left(\int_{0}^{1} \mathbf{F}_{3}(r) e^{\tau b r} \mathrm{~d} r+\mathbf{E}^{\prime} \times \boldsymbol{\nu}\right)\right)\right) \mathrm{d} \mathbf{x} .
\end{aligned}
$$

The latter two integrals rewrite as

$$
\begin{aligned}
& b \gamma_{1} \int_{\Gamma}(\tilde{\mathbf{E}} \times \boldsymbol{\nu}) \cdot\left(\mathbf{g}(\mathbf{E} \times \boldsymbol{\nu})-\mathbf{g}\left(\mathbf{E}^{\prime} \times \boldsymbol{\nu}\right)\right) \mathrm{d} \mathbf{x} \\
& +b \gamma_{2} \int_{\Gamma}(\tilde{\mathbf{E}} \times \boldsymbol{\nu}) \cdot\left(\mathbf{g}\left(e^{-\tau b}\left(\int_{0}^{1} \mathbf{F}_{3}(r) e^{\tau b r} \mathrm{~d} r+\mathbf{E} \times \boldsymbol{\nu}\right)\right)-\mathbf{g}\left(e^{-\tau b}\left(\int_{0}^{1} \mathbf{F}_{3}(r) e^{\tau b r} \mathrm{~d} r+\mathbf{E}^{\prime} \times \boldsymbol{\nu}\right)\right)\right) \mathrm{d} \mathbf{x} .
\end{aligned}
$$


Utilizing Assumption 2.2, we obtain

$$
b \gamma_{1} \int_{\Gamma}(\tilde{\mathbf{E}} \times \boldsymbol{\nu}) \cdot\left(\mathbf{g}(\mathbf{E} \times \boldsymbol{\nu})-\mathbf{g}\left(\mathbf{E}^{\prime} \times \boldsymbol{\nu}\right)\right) \mathrm{d} \mathbf{x} \geq b \gamma_{1} \int_{\Gamma} c_{1}|\tilde{\mathbf{E}} \times \boldsymbol{\nu}|^{2} \mathrm{~d} \mathbf{x}
$$

and

$$
\begin{aligned}
& b \gamma_{2} \int_{\Gamma}(\tilde{\mathbf{E}} \times \boldsymbol{\nu}) \cdot\left(\mathbf{g}\left(e^{-\tau b}\left(\int_{0}^{1} \mathbf{F}_{3}(r) e^{\tau b r} \mathrm{~d} r+\mathbf{E} \times \boldsymbol{\nu}\right)\right)-\mathbf{g}\left(e^{-\tau b}\left(\int_{0}^{1} \mathbf{F}_{3}(r) e^{\tau b r} \mathrm{~d} r+\mathbf{E}^{\prime} \times \boldsymbol{\nu}\right)\right)\right) \mathrm{d} \mathbf{x} \\
& \geq b \gamma_{2} e^{\tau b} \int_{\Gamma} c_{1}\left|e^{-\tau b}(\tilde{\mathbf{E}} \times \boldsymbol{\nu})\right|^{2} \mathrm{~d} \mathbf{x}=b \gamma_{2} c_{1} e^{-\tau b} \int_{\Gamma}|(\tilde{\mathbf{E}} \times \boldsymbol{\nu})|^{2} \mathrm{~d} \mathbf{x} .
\end{aligned}
$$

Hence,

$$
\begin{aligned}
\left\langle\mathcal{B} \mathbf{E}-\mathcal{B} \mathbf{E}^{\prime}, \mathbf{E}-\mathbf{E}^{\prime}\right\rangle_{W_{\varepsilon}^{\prime} \times W_{\varepsilon}} & \geq \int_{G} b^{2} \varepsilon \tilde{\mathbf{E}} \cdot \tilde{\mathbf{E}}+\boldsymbol{\mu}^{-1}|\operatorname{curl} \tilde{\mathbf{E}}|^{2}+s|\operatorname{div}(\varepsilon \tilde{\mathbf{E}})|^{2} \mathrm{~d} \mathbf{x} \\
& +b c_{1}\left(\gamma_{1}+e^{-\tau b} \gamma_{2}\right) \int_{\Gamma} c_{1}|\tilde{\mathbf{E}} \times \boldsymbol{\nu}|^{2} \mathrm{~d} \mathbf{x} \\
& \geq c^{*}\left\|\mathbf{E}-\mathbf{E}^{\prime}\right\|_{W_{\varepsilon}}^{2}
\end{aligned}
$$

for some positive $c^{*}$.

Hemicontinuity: For any $\mathbf{E}, \mathbf{E}^{\prime} \in W_{\boldsymbol{\varepsilon}}$, we can write

$$
\begin{aligned}
\langle\mathcal{B}(\mathbf{E} & \left.\left.+t \mathbf{E}^{\prime}\right), \mathbf{E}^{\prime}\right\rangle_{W_{\varepsilon}^{\prime} \times W_{\varepsilon}}=\mathfrak{a}\left(\mathbf{E}+t \mathbf{E}^{\prime}, \mathbf{E}^{\prime}\right) \\
& =\int_{G} b^{2} \varepsilon\left(\mathbf{E}+t \mathbf{E}^{\prime}\right) \cdot \mathbf{E}^{\prime}+\boldsymbol{\mu}^{-1} \operatorname{curl}\left(\mathbf{E}+t \mathbf{E}^{\prime}\right) \cdot \operatorname{curl} \mathbf{E}^{\prime}+s \operatorname{div}\left(\varepsilon\left(\mathbf{E}+t \mathbf{E}^{\prime}\right)\right) \operatorname{div}\left(\varepsilon \mathbf{E}^{\prime}\right) \mathrm{d} \mathbf{x} \\
& +b \gamma_{1} \int_{\Gamma}\left(\mathbf{E}^{\prime} \times \boldsymbol{\nu}\right) \cdot \mathbf{g}\left(\left(\mathbf{E}+t \mathbf{E}^{\prime}\right) \times \boldsymbol{\nu}\right) \mathrm{d} \mathbf{x} \\
& +b \gamma_{2} \int_{\Gamma}\left(\mathbf{E}^{\prime} \times \boldsymbol{\nu}\right) \cdot \mathbf{g}\left(e^{-\tau b}\left(\int_{0}^{1} \mathbf{F}_{3}(r) e^{\tau b r} \mathrm{~d} r+\left(\mathbf{E}+t \mathbf{E}^{\prime}\right) \times \boldsymbol{\nu}\right)\right) \mathrm{d} \mathbf{x} .
\end{aligned}
$$

On the strength of Assumption 2.2, we get the continuity of $\mathbf{g}(\cdot)$. Now, by virtue of Equation 2.25, the continuity of $t \mapsto\left\langle\mathcal{B}\left(\mathbf{E}+t \mathbf{E}^{\prime}\right), \mathbf{E}^{\prime}\right\rangle_{W_{\varepsilon}^{\prime} \times W_{\varepsilon}}$ follows.

Boundedness: Suppose $\|\mathbf{E}\|_{W_{\varepsilon}} \leq c$. Then,

$$
\begin{aligned}
\left|\left\langle\mathcal{B} \mathbf{E}, \mathbf{E}^{\prime}\right\rangle_{W_{\varepsilon}^{\prime} \times W_{\varepsilon}}\right| & =\left|\mathfrak{a}\left(\mathbf{E}, \mathbf{E}^{\prime}\right)\right| \\
& \leq \int_{G} b^{2}\left|\varepsilon \mathbf{E} \cdot \mathbf{E}^{\prime}\right|+\left|\boldsymbol{\mu}^{-1} \operatorname{curl} \mathbf{E} \cdot \operatorname{curl} \mathbf{E}^{\prime}\right|+s\left|\operatorname{div}(\varepsilon \mathbf{E}) \operatorname{div}\left(\varepsilon \mathbf{E}^{\prime}\right)\right| \mathrm{d} \mathbf{x} \\
& +b \gamma_{1} \int_{\Gamma}\left|\left(\mathbf{E}^{\prime} \times \boldsymbol{\nu}\right) \cdot \mathbf{g}(\mathbf{E} \times \boldsymbol{\nu})\right| \mathrm{d} \mathbf{x} \\
& +b \gamma_{2} \int_{\Gamma}\left|\left(\mathbf{E}^{\prime} \times \boldsymbol{\nu}\right) \cdot \mathbf{g}\left(e^{-\tau b}\left(\int_{0}^{1} \mathbf{F}_{3}(r) e^{\tau b r} \mathrm{~d} r+\mathbf{E} \times \boldsymbol{\nu}\right)\right)\right| \mathrm{d} \mathbf{x} .
\end{aligned}
$$

Using Cauchy \& Schwarz' inequality and Assumption 2.2, we estimate

$$
\begin{aligned}
b \gamma_{1} \int_{\Gamma} \mid\left(\mathbf{E}^{\prime} \times \boldsymbol{\nu}\right) & \cdot \mathbf{g}(\mathbf{E} \times \boldsymbol{\nu}) \mid \mathrm{d} \mathbf{x} \leq b \gamma_{1}\left(\int_{\Gamma}\left|\mathbf{E}^{\prime} \times \boldsymbol{\nu}\right|^{2} \mathrm{~d} \mathbf{x} \int_{\Gamma}|\mathbf{g}(\mathbf{E} \times \boldsymbol{\nu})|^{2} \mathrm{~d} \mathbf{x}\right)^{1 / 2} \\
& \leq b \gamma_{1} c_{2}\left(\int_{\Gamma}\left|\mathbf{E}^{\prime} \times \boldsymbol{\nu}\right|^{2} \mathrm{~d} \mathbf{x} \int_{\Gamma}|\mathbf{E} \times \boldsymbol{\nu}|^{2} \mathrm{~d} \mathbf{x}\right)^{1 / 2}
\end{aligned}
$$




$$
\begin{aligned}
& =b \gamma_{1} c_{2}\left\|\mathbf{E}^{\prime} \times \boldsymbol{\nu}\right\|_{\left(L^{2}(\Gamma)\right)^{3}}\|\mathbf{E} \times \boldsymbol{\nu}\|_{\left(L^{2}(\Gamma)\right)^{3}} \\
& \leq b \gamma_{1} c_{2}\left\|\mathbf{E}^{\prime}\right\|_{W_{\boldsymbol{\varepsilon}}}\|\mathbf{E}\|_{W_{\boldsymbol{\varepsilon}}} \\
& \leq b \gamma_{1} c_{2} c\left\|\mathbf{E}^{\prime}\right\|_{W_{\boldsymbol{\varepsilon}}}
\end{aligned}
$$

and

$$
\begin{aligned}
b \gamma_{2} \int_{\Gamma} \mid & \left(\mathbf{E}^{\prime} \times \boldsymbol{\nu}\right) \cdot \mathbf{g}\left(e^{-\tau b}\left(\int_{0}^{1} \mathbf{F}_{3}(r) e^{\tau b r} \mathrm{~d} r+\mathbf{E} \times \boldsymbol{\nu}\right)\right) \mid \mathrm{d} \mathbf{x} \\
& \leq b \gamma_{2} c_{2}\left(\int_{\Gamma}\left|\mathbf{E}^{\prime} \times \boldsymbol{\nu}\right|^{2} \mathrm{~d} \mathbf{x} \int_{\Gamma}\left|e^{-\tau b}\left(\int_{0}^{1} \mathbf{F}_{3}(r) e^{\tau b r} \mathrm{~d} r+\mathbf{E} \times \boldsymbol{\nu}\right)\right|^{2} \mathrm{~d} \mathbf{x}\right)^{1 / 2} \\
& \leq b \gamma_{2} c_{2} e^{-\tau b}\left\|\mathbf{E}^{\prime} \times \boldsymbol{\nu}\right\|_{\left(L^{2}(\Gamma)\right)^{3}}\left(\int_{\Gamma} 2\left(\int_{0}^{1} \mathbf{F}_{3}(r) e^{\tau b r} \mathrm{~d} r\right)^{2}+2|\mathbf{E} \times \boldsymbol{\nu}|^{2} \mathrm{~d} \mathbf{x}\right)^{1 / 2} \\
& \leq 2 b \gamma_{2} c_{2} e^{-\tau b}\left\|\mathbf{E}^{\prime}\right\|_{W_{\boldsymbol{\varepsilon}}}\left(\left\|\mathcal{I} \mathbf{F}_{3}\right\|_{\left(L^{2}(\Gamma)\right)^{3}}+\|\mathbf{E} \times \boldsymbol{\nu}\|_{\left(L^{2}(\Gamma)\right)^{3}}\right) \\
& \leq 2 b \gamma_{2} c_{2} e^{-\tau b}\left\|\mathbf{E}^{\prime}\right\|_{W_{\boldsymbol{\varepsilon}}}\left(\left\|\mathcal{I} \mathbf{F}_{3}\right\|_{\left(L^{2}(\Gamma)\right)^{3}}+c\right),
\end{aligned}
$$

where $\mathcal{I} \mathbf{F}_{3}=\int_{0}^{1} \mathbf{F}_{3}(r) e^{\tau b r} \mathrm{~d} r$. Therefore, $\left|\left\langle\mathcal{B} \mathbf{E}, \mathbf{E}^{\prime}\right\rangle_{W_{\varepsilon}^{\prime} \times W_{\varepsilon}}\right| \leq c^{*}\left\|\mathbf{E}^{\prime}\right\|_{W_{\varepsilon}}$ for a suitable $c^{*}$. Thus, $\|\mathcal{B} \mathbf{E}\|_{W_{\varepsilon}^{\prime}} \leq c^{*}$ and the conclusion follows.

In summary, $\mathcal{B}$ is surjective and the problem 2.20 possesses a (weak) solution. Since $\mathcal{B}$ is strongly monotone, the solution is unique.

Strongness of solution: We now prove the (weak) solution $\mathbf{E} \in W_{\boldsymbol{\varepsilon}}$ to Equation (2.20) along with corresponding $\mathbf{H}, \mathbf{Z}$ satisfy Equation 2.10 .

First, we show that $\operatorname{div}(\varepsilon \mathbf{E})=0$. Following [9], consider the set

$$
D=\left\{\varphi \in H_{0}^{1}(G) \mid \operatorname{div}(\varepsilon \nabla \varphi) \in L^{2}(G)\right\} .
$$

Letting $\mathbf{E}^{\prime}=\nabla \varphi$ for arbitrary, but fixed $\varphi \in D$, we can rewrite Equation 2.20 as

$$
\int_{G} b^{2} \varepsilon \mathbf{E} \cdot \nabla \varphi+s \operatorname{div}(\varepsilon \mathbf{E}) \operatorname{div}(\varepsilon \nabla \varphi) \mathrm{d} \mathbf{x}=\int_{G} b \varepsilon \mathbf{F}_{1} \cdot \nabla \varphi \mathrm{d} \mathbf{x} \text { for any } \varphi \in D .
$$

Using Green's formula, we get

$$
\int_{G}-b^{2} \operatorname{div}(\varepsilon \mathbf{E}) \varphi+s \operatorname{div}(\varepsilon \mathbf{E}) \operatorname{div}(\varepsilon \nabla \varphi) \mathrm{d} \mathbf{x}=-\int_{G} b \operatorname{div}\left(\varepsilon \mathbf{F}_{1}\right) \varphi \mathrm{d} \mathbf{x} \text { for any } \varphi \in D .
$$

Since $\left(\mathbf{F}_{1}, \mathbf{F}_{2}, \mathbf{F}_{3}\right)^{T} \in \mathscr{H}$, it follows that $\mathbf{F}_{1} \in H\left(\operatorname{div}_{\boldsymbol{\varepsilon}} 0, G\right)$. Thus, the latter integral in Equation (2.27) vanishes. Hence,

$$
\int_{G} \operatorname{div}(\varepsilon \mathbf{E})\left(-b^{2} \varphi+s \operatorname{div}(\varepsilon \nabla \varphi)\right) \mathrm{d} \mathbf{x}=0 \text { for any } \varphi \in D .
$$

Since the spectrum of $\operatorname{div}(\varepsilon \nabla \cdot)$ with homogeneous Dirichlet boundary conditions is discrete, there exists a positive number $s$ such that $b^{2} / s$ belongs to the resolvent set. Then, from Equation 2.28 , we conclude that $\operatorname{div}(\varepsilon \mathbf{E})=0$ holds strongly in $G$.

Therefore, Equation 2.20 becomes

$$
\int_{G} b^{2} \varepsilon \mathbf{E} \cdot \mathbf{E}^{\prime}+\boldsymbol{\mu}^{-1} \operatorname{curl} \mathbf{E} \cdot \operatorname{curl} \mathbf{E}^{\prime}+b \int_{\Gamma}\left(\mathbf{E}^{\prime} \times \boldsymbol{\nu}\right) \cdot\left(\gamma_{1} \mathbf{g}\left(\left.\mathbf{Z}\right|_{s=0}\right)+\gamma_{2} \mathbf{g}\left(\left.\mathbf{Z}\right|_{s=1}\right)\right) \mathrm{d} \mathbf{x}
$$




$$
=\int_{G} b \varepsilon \mathbf{F}_{1} \cdot \mathbf{E}^{\prime}+\mathbf{F}_{2} \cdot \operatorname{curl} \mathbf{E}^{\prime} \mathrm{d} \mathbf{x} \text { for any } \mathbf{E}^{\prime} \in W_{\varepsilon}
$$

Recalling the definition of $\mathbf{H}$ from Equation (2.14) and applying Green's formula to Equation 2.29 , we arrive at

$$
\begin{aligned}
\int_{G} \varepsilon b \mathbf{E} \cdot \mathbf{E}^{\prime}+\mathbf{H} \cdot \operatorname{curl} \mathbf{E}^{\prime} \mathrm{d} \mathbf{x} & +\int_{\Gamma}\left(\mathbf{E}^{\prime} \times \boldsymbol{\nu}\right) \cdot\left(\gamma_{1} \mathbf{g}\left(\left.\mathbf{Z}\right|_{s=0}\right)+\gamma_{2} \mathbf{g}\left(\left.\mathbf{Z}\right|_{s=1}\right)\right) \mathrm{d} \mathbf{x} \\
& =\int_{G} \varepsilon \mathbf{F}_{1} \cdot \mathbf{E}^{\prime} \mathrm{d} \mathbf{x} \text { for any } \mathbf{E}^{\prime} \in W_{\varepsilon} .
\end{aligned}
$$

Choosing $\mathbf{E}^{\prime}=P_{\boldsymbol{\varepsilon}} \chi$ with $\chi \in(\mathcal{D}(G))^{3}$, we get

$$
\int_{G} \varepsilon b \mathbf{E} \cdot P_{\varepsilon} \chi+\mathbf{H} \cdot \operatorname{curl}\left(P_{\varepsilon} \chi\right) \mathrm{d} \mathbf{x}=\int_{G} \varepsilon \mathbf{F}_{1} \cdot P_{\varepsilon} \chi \mathrm{d} \mathbf{x} \text { for any } \chi \in(\mathcal{D}(G))^{3}
$$

or, after using Green's formula,

$$
\int_{G}(\varepsilon b \mathbf{E}-\operatorname{curl} \mathbf{H}) \cdot P_{\varepsilon} \chi \mathrm{d} \mathbf{x}=\int_{G} \varepsilon \mathbf{F}_{1} \cdot P_{\varepsilon} \chi \mathrm{d} \mathbf{x} \text { for any } \chi \in(\mathcal{D}(G))^{3} .
$$

Since $P_{\boldsymbol{\varepsilon}}(\mathcal{D}(G))^{3}$ is dense in $H\left(\operatorname{div}_{\boldsymbol{\varepsilon}} 0, G\right)$, there identity

$$
\varepsilon b \mathbf{E}-\operatorname{curl} \mathbf{H}=\varepsilon \mathbf{F}_{1}
$$

follows in the strong sense.

Choosing $\mathbf{E}^{\prime}=P_{\varepsilon} \chi$ with $\chi \in\left(C^{\infty}(G)\right)^{3}$, we get

$$
\begin{aligned}
\int_{G} \varepsilon b \mathbf{E} \cdot P_{\varepsilon} \chi & +\mathbf{H} \cdot \operatorname{curl}\left(P_{\varepsilon} \chi\right) \mathrm{d} \mathbf{x}+\int_{\Gamma}\left(P_{\varepsilon} \chi \times \boldsymbol{\nu}\right) \cdot\left(\gamma_{1} \mathbf{g}\left(\left.\mathbf{Z}\right|_{s=0}\right)+\gamma_{2} \mathbf{g}\left(\left.\mathbf{Z}\right|_{s=1}\right)\right) \mathrm{d} \mathbf{x} \\
& =\int_{G} \varepsilon \mathbf{F}_{1} \cdot P_{\varepsilon} \chi \mathrm{d} \mathbf{x} \text { for all } \chi \in(\mathcal{D}(G))^{3} .
\end{aligned}
$$

Using Equation (2.30), Lemma 2.6 and Green's formula, we finally conclude

$$
\int_{\Gamma}-(\mathbf{H} \times \boldsymbol{\nu}) \cdot \boldsymbol{\chi} \mathrm{d} \mathbf{x}+\int_{\Gamma}\left(\boldsymbol{\nu} \times\left(\gamma_{1} \mathbf{g}\left(\left.\mathbf{Z}\right|_{s=0}\right)+\gamma_{2} \mathbf{g}\left(\left.\mathbf{Z}\right|_{s=1}\right)\right)\right) \cdot \chi \mathrm{d} \mathbf{x}=0
$$

for all $\boldsymbol{\chi} \in(\mathcal{D}(G))^{3}$. Thus, we have $-\mathbf{H} \times \boldsymbol{\nu}-\left(\gamma_{1} \mathbf{g}\left(\left.\mathbf{Z}\right|_{s=0}\right)+\gamma_{2} \mathbf{g}\left(\left.\mathbf{Z}\right|_{s=1}\right)\right) \times \boldsymbol{\nu}=0$ in the strong sense. Therefore, $(\mathbf{E}, \mathbf{H}, \mathbf{Z})^{T} \in D(\mathscr{A})$ and Equation $(2.10)$ is satisfied.

Theorem 2.8. Under Assumptions 2.1 and 2.2, suppose $\mathbf{V}^{0} \in \mathscr{H}$. Then, Equation (2.1) possesses a unique global mild solution

$$
\mathbf{V} \in C^{0}([0, \infty), \mathscr{H}) .
$$

If, moreover, $\mathbf{V}^{0} \in D(\mathscr{A})$, the mild solution $\mathbf{V}$ is a strong solution satisfying

$$
\mathbf{V} \in W_{\mathrm{loc}}^{1, \infty}(0, \infty ; \mathscr{H}) \cap L_{\mathrm{loc}}^{\infty}(0, \infty ; D(\mathscr{A})) .
$$

Proof. Since the operator $C$ id $+\mathcal{A}$ is maximally monotone for a sufficiently large $C>0$, using [1, Corollary 4.1], any initial value $\mathbf{V}^{0} \in \overline{D(\mathscr{A})}$ admits a unique mild solution. By virtue of Lemma 2.5. this remains true for $\mathbf{V}^{0} \in \mathscr{H}$. As for the strong solution, [1, Theorem 4.5] applies. 


\section{$3 \quad$ Exponential Stability}

Our thrust is to prove the exponential stability for Equations $(1.9)-(1.13)$. To this end, we consider the "natural energy" functional

$$
E(t):=\frac{1}{2}\|\mathbf{V}\|_{\mathscr{H}}^{2} \equiv \frac{1}{2} \int_{G}|\mathbf{E}(t, \cdot)|^{2} \mathrm{~d} \mathbf{x}+\frac{1}{2} \int_{G}|\mathbf{H}(t, \cdot)|^{2} \mathrm{~d} \mathbf{x}+\tau \int_{0}^{1} \int_{\Gamma}|\mathbf{E}(t-\tau s, \mathbf{x}) \times \boldsymbol{\nu}|^{2} \mathrm{~d} \mathbf{x} \mathrm{d} s
$$

In the following, we apply a combination of Rellich's multiplier techniques developed for boundary control problems along with Lyapunov's techniques for delay differential equations in the spirit of [13].

For $\mathbf{x}_{0} \in \mathbb{R}^{3}$, consider the vector field $\mathbf{m}(\mathbf{x}):=\mathbf{x}-\mathbf{x}_{0}$.

Assumption 3.1 (Regularity and geometric conditions). Suppose the following conditions are satisfied:

1. $G$ is a bounded $C^{2}$-domain.

2. $G$ is strictly star-shaped with respect to $\mathbf{x}_{0} \in G$, i.e.,

$$
\mathbf{m}(\mathbf{x}) \cdot \boldsymbol{\nu}(\mathbf{x})>0 \text { for } \mathbf{x} \in \Gamma .
$$

3. $\varepsilon, \boldsymbol{\mu} \in C^{1}\left(\bar{G}, \mathbb{R}^{3 \times 3}\right)$.

4. There exists a constant $d_{1}>0$ such that

$$
\varepsilon+(\mathbf{m} \cdot \nabla) \varepsilon \geq d_{1} \varepsilon \quad \text { and } \quad \boldsymbol{\mu}+(\mathbf{m} \cdot \nabla) \boldsymbol{\mu} \geq d_{1} \boldsymbol{\mu} \text { in } \bar{G},
$$

Remark 3.2. Inequalities (3.2) are mathematical assumptions about the physical nature of the medium (cf. [6]). Similar conditions are imposed in [10, 15], etc. In case both $\boldsymbol{\varepsilon}$ and $\boldsymbol{\mu}$ are scalar and constant (or "nearly" constant), this corresponds to the "strict star-shapedness" with respect to $\mathbf{x}_{0}$ (see, e.g., 14, p. 2]). In particular, all convex domains are strictly star-shaped. Hence, the geometry class is non-trivial.

Consider a new functional

$$
E_{\xi}(t):=\frac{1}{2} \int_{G}|\mathbf{E}(t, \cdot)|^{2} \mathrm{~d} \mathbf{x}+\frac{1}{2} \int_{G}|\mathbf{H}(t, \cdot)|^{2} \mathrm{~d} \mathbf{x}+\xi \tau \int_{0}^{1} \int_{\Gamma}|\mathbf{E}(t-\tau s, \mathbf{x}) \times \boldsymbol{\nu}|^{2} \mathrm{~d} \mathbf{x} \mathrm{d} s,
$$

where $\xi$ is a positive number such that

$$
\gamma_{1} c_{1}-\frac{\gamma_{2} c_{2}}{2}>\xi>\frac{\gamma_{2} c_{2}}{2}
$$

Obviously, $\xi$ exists if $\gamma_{1} c_{1}>\gamma_{2} c_{2}$.

Lemma 3.3. Suppose $\gamma_{1} c_{1}>\gamma_{2} c_{2}$. Then, there exist positive numbers $c_{1}^{E}, c_{2}^{E}$ such that for all $t_{2}>t_{1} \geq 0$ the following inequality holds

$$
-\left.c_{1}^{E} \int_{t_{1}}^{t_{2}} \int_{\Gamma} \mathbf{Z}\right|_{s=0} ^{2}+\left.\mathbf{Z}\right|_{s=1} ^{2} \mathrm{~d} \mathbf{x} \mathrm{d} t \geq E_{\xi}\left(t_{2}\right)-E_{\xi}\left(t_{1}\right) \geq-\left.c_{2}^{E} \int_{t_{1}}^{t_{2}} \int_{\Gamma} \mathbf{Z}\right|_{s=0} ^{2}+\left.\mathbf{Z}\right|_{s=1} ^{2} \mathrm{~d} \mathbf{x} \mathrm{d} t
$$

where $(\mathbf{E}, \mathbf{H}, \mathbf{Z})^{T}$ is a strong solution of Equation 2.1). 
Proof. Similar to [9, Lemma 2.7], multiplying Equations $\sqrt{1.9}$ and $\sqrt{1.10}$ in $L^{2}\left(0, T ;\left(L^{2}(G)\right)^{3}\right)$ with $\mathbf{E}$ and $\mathbf{H}$, respectively, integrating by parts and using the boundary condition from Equation (1.11), we get

$$
\begin{aligned}
E_{\xi}\left(t_{2}\right)-E_{\xi}\left(t_{1}\right) & =-\int_{t_{1}}^{t_{2}} \int_{\Gamma}\left(\gamma_{1} \mathbf{g}\left(\left.\mathbf{Z}\right|_{s=0}\right)+\gamma_{2} \mathbf{g}\left(\left.\mathbf{Z}\right|_{s=1}\right)\right) \cdot(\mathbf{E}(t, \cdot) \times \boldsymbol{\nu}) \mathrm{d} \mathbf{x} \mathrm{d} t \\
& +\xi \tau \int_{t_{1}}^{t_{2}} \int_{0}^{1} \int_{\Gamma} 2(\mathbf{E}(t-\tau s, \cdot) \times \boldsymbol{\nu}) \cdot \partial_{t}(\mathbf{E}(t-\tau s, \cdot) \times \boldsymbol{\nu}) \mathrm{d} \mathbf{x} \mathrm{d} s \mathrm{~d} t
\end{aligned}
$$

Recalling

$$
\mathbf{Z}(t, s, \cdot)=\mathbf{E}(t-\tau s, \cdot) \times \boldsymbol{\nu} \text { for } s \in[0,1]
$$

and following [13], we obtain

$$
\tau \partial_{t} \mathbf{Z}(t, s, \cdot)+\partial_{s} \mathbf{Z}(t, s, \cdot)=\mathbf{0} \text { for }(t, s) \in(0, \infty) \times(0,1) .
$$

Therefore,

$$
\begin{aligned}
\xi \tau \int_{0}^{1} \int_{\Gamma} 2(\mathbf{E}(t-\tau s, \cdot) \times \boldsymbol{\nu}) & \cdot \partial_{t}(\mathbf{E}(t-\tau s, \cdot) \times \boldsymbol{\nu}) \mathrm{d} \mathbf{x} \mathrm{d} s \\
& =-\xi \int_{0}^{1} \int_{\Gamma} 2(\mathbf{E}(t-\tau s, \cdot) \times \boldsymbol{\nu}) \cdot \partial_{s}(\mathbf{E}(t-\tau s, \cdot) \times \boldsymbol{\nu}) \mathrm{d} \mathbf{x} \mathrm{d} s \\
& =-\xi \int_{0}^{1} \int_{\Gamma} \partial_{s}|\mathbf{E}(t-\tau s, \cdot) \times \boldsymbol{\nu}|^{2} \mathrm{~d} \mathbf{x} \mathrm{d} s \\
& =-\left.\xi \int_{\Gamma}|\mathbf{E}(t-\tau s, \cdot) \times \boldsymbol{\nu}|^{2}\right|_{s=0} ^{s=1} \mathrm{~d} \mathbf{x} \\
& =\left.\xi \int_{\Gamma} \mathbf{Z}\right|_{s=0} ^{2}-\left.\mathbf{Z}\right|_{s=1} ^{2} \mathrm{~d} \mathbf{x} .
\end{aligned}
$$

After plugging the latter identity into Equation (3.5), we arrive at

$$
\begin{aligned}
E_{\xi}\left(t_{2}\right)-E_{\xi}\left(t_{1}\right) & =-\left.\int_{t_{1}}^{t_{2}} \int_{\Gamma}\left(\gamma_{1} \mathbf{g}\left(\left.\mathbf{Z}\right|_{s=0}\right)+\gamma_{2} \mathbf{g}\left(\left.\mathbf{Z}\right|_{s=1}\right)\right) \cdot \mathbf{Z}\right|_{s=0} \mathrm{~d} \mathbf{x} \mathrm{d} t \\
& +\left.\xi \int_{t_{1}}^{t_{2}} \int_{\Gamma} \mathbf{Z}\right|_{s=0} ^{2}-\left.\mathbf{Z}\right|_{s=1} ^{2} \mathrm{~d} \mathbf{x} \mathrm{d} t .
\end{aligned}
$$

Using Assumption 2.2 and Young's inequality, we get

$$
\begin{aligned}
\left.\int_{\Gamma} \mathbf{g}\left(\left.\mathbf{Z}\right|_{s=0}\right) \cdot \mathbf{Z}\right|_{s=0} \mathrm{~d} \mathbf{x} & \geq\left. c_{1} \int_{\Gamma} \mathbf{Z}\right|_{s=0} ^{2} \mathrm{~d} \mathbf{x} \text { and } \\
\left.\int_{\Gamma} \mathbf{g}\left(\left.\mathbf{Z}\right|_{s=1}\right) \cdot \mathbf{Z}\right|_{s=0} \mathrm{~d} \mathbf{x} & \geq-\left.c_{2} \int_{\Gamma}|\mathbf{Z}|_{s=1} \cdot \mathbf{Z}\right|_{s=0} \mid \mathrm{d} \mathbf{x} \\
& \geq-\left.\frac{c_{2}}{2} \int_{\Gamma} \mathbf{Z}\right|_{s=1} ^{2} \mathrm{~d} \mathbf{x}-\left.\frac{c_{2}}{2} \int_{\Gamma} \mathbf{Z}\right|_{s=0} ^{2} \mathrm{~d} \mathbf{x} .
\end{aligned}
$$

Then, Equation (3.6) can further be estimated as follows:

$$
E_{\xi}\left(t_{2}\right)-E_{\xi}\left(t_{1}\right) \leq \int_{t_{1}}^{t_{2}}\left(-\left.\gamma_{1} c_{1} \int_{\Gamma} \mathbf{Z}\right|_{s=0} ^{2} \mathrm{~d} \mathbf{x}+\left.\frac{\gamma_{2} c_{2}}{2} \int_{\Gamma} \mathbf{Z}\right|_{s=1} ^{2} \mathrm{~d} \mathbf{x}+\left.\frac{\gamma_{2} c_{2}}{2} \int_{\Gamma} \mathbf{Z}\right|_{s=0} ^{2} \mathrm{~d} \mathbf{x}\right) \mathrm{d} t
$$




$$
\begin{aligned}
& +\left.\xi \int_{t_{1}}^{t_{2}} \int_{\Gamma} \mathbf{Z}\right|_{s=0} ^{2}-\left.\mathbf{Z}\right|_{s=1} ^{2} \mathrm{~d} \mathbf{x} \mathrm{d} t \\
& =-\left.\left(\gamma_{1} c_{1}-\frac{\gamma_{2} c_{2}}{2}-\xi\right) \int_{t_{1}}^{t_{2}} \int_{\Gamma} \mathbf{Z}\right|_{s=0} ^{2} \mathrm{~d} \mathbf{x} \mathrm{d} t-\left.\left(\xi-\frac{\gamma_{2} c_{2}}{2}\right) \int_{t_{1}}^{t_{2}} \int_{\Gamma} \mathbf{Z}\right|_{s=1} ^{2} \mathrm{~d} \mathbf{x} \mathrm{d} t .
\end{aligned}
$$

Since $\xi$ is selected to satisfy Equation (3.3), we arrive at

$$
E_{\xi}\left(t_{2}\right)-E_{\xi}\left(t_{1}\right) \leq-\left.c_{1}^{E} \int_{t_{1}}^{t_{2}} \int_{\Gamma} \mathbf{Z}\right|_{s=0} ^{2}+\left.\mathbf{Z}\right|_{s=1} ^{2} \mathrm{~d} \mathbf{x} \mathrm{d} t
$$

On the other hand,

$$
\begin{aligned}
\left|\int_{\Gamma} \mathbf{g}\left(\left.\mathbf{Z}\right|_{s=0}\right) \cdot \mathbf{Z}\right|_{s=0} \mathrm{~d} \mathbf{x} \mid & \leq\left. c_{2} \int_{\Gamma} \mathbf{Z}\right|_{s=0} ^{2} \mathrm{~d} \mathbf{x} \text { and } \\
\left|\int_{\Gamma} \mathbf{g}\left(\left.\mathbf{Z}\right|_{s=1}\right) \cdot \mathbf{Z}\right|_{s=0} \mathrm{~d} \mathbf{x} \mid & \leq\left. c_{2} \int_{\Gamma}|\mathbf{Z}|_{s=1} \cdot \mathbf{Z}\right|_{s=0} \mid \mathrm{d} \mathbf{x} \\
& \leq\left.\frac{c_{2}}{2} \int_{\Gamma} \mathbf{Z}\right|_{s=1} ^{2} \mathrm{~d} \mathbf{x}+\left.\frac{c_{2}}{2} \int_{\Gamma} \mathbf{Z}\right|_{s=0} ^{2} \mathrm{~d} \mathbf{x} .
\end{aligned}
$$

Thus,

$$
\begin{aligned}
E_{\xi}\left(t_{2}\right)-E_{\xi}\left(t_{1}\right) & \geq-\left|\int_{t_{1}}^{t_{2}} \int_{\Gamma}\left(\gamma_{1} \mathbf{g}\left(\left.\mathbf{Z}\right|_{s=0}\right)+\gamma_{2} \mathbf{g}\left(\left.\mathbf{Z}\right|_{s=1}\right)\right) \cdot \mathbf{Z}\right|_{s=0} \mathrm{~d} \mathbf{x} \mathrm{d} t \mid \\
& -\xi\left|\int_{t_{1}}^{t_{2}} \int_{\Gamma} \mathbf{Z}\right|_{s=0}^{2}-\left.\mathbf{Z}\right|_{s=1} ^{2} \mathrm{~d} \mathbf{x} \mathrm{d} t \mid \\
& \geq \int_{t_{1}}^{t_{2}}\left(-\left.\gamma_{1} c_{2} \int_{\Gamma} \mathbf{Z}\right|_{s=0} ^{2} \mathrm{~d} \mathbf{x}-\left.\frac{\gamma_{2} c_{2}}{2} \int_{\Gamma} \mathbf{Z}\right|_{s=1} ^{2} \mathrm{~d} \mathbf{x}-\left.\frac{\gamma_{2} c_{2}}{2} \int_{\Gamma} \mathbf{Z}\right|_{s=0} ^{2} \mathrm{~d} \mathbf{x}\right) \mathrm{d} t \\
& -\left.\xi \int_{t_{1}}^{t_{2}} \int_{\Gamma} \mathbf{Z}\right|_{s=0} ^{2} \mathrm{~d} \mathbf{x} \mathrm{d} t-\left.\xi \int_{t_{1}}^{t_{2}} \int_{\Gamma} \mathbf{Z}\right|_{s=1} ^{2} \mathrm{~d} \mathbf{x} \mathrm{d} t \\
& \geq-\left.c_{2}^{E} \int_{t_{1}}^{t_{2}} \int_{\Gamma} \mathbf{Z}\right|_{s=0} ^{2}+\left.\mathbf{Z}\right|_{s=1} ^{2} \mathrm{~d} \mathbf{x} \mathrm{d} t
\end{aligned}
$$

which finishes the proof.

Lemma 3.4. There exist positive numbers $c, c_{T}$ such that the estimate

$$
\int_{0}^{T} E_{\xi}(t) \mathrm{d} t \leq c\left(E_{\xi}(0)+E_{\xi}(T)\right)+\left.c_{T} \int_{0}^{T} \int_{\Gamma} \mathbf{Z}\right|_{s=0} ^{2}+\left.\mathbf{Z}\right|_{s=1} ^{2} \mathrm{~d} \mathbf{x} \mathrm{d} t
$$

holds true for every $T>0$ along any strong solution $(\mathbf{E}, \mathbf{H}, \mathbf{Z})^{T}$ of Equation 2.1).

Proof. Similar to [6, Section 3.1, pp. 193-195], using Rellich's multipliers $\mathbf{m} \times(\varepsilon \mathbf{E})$ and $\mathbf{m} \times(\boldsymbol{\mu H})$, we obtain

$$
\begin{aligned}
\frac{1}{2} \int_{0}^{T} \int_{G} & (\varepsilon+(\mathbf{m} \cdot \nabla) \varepsilon) \mathbf{E} \cdot \mathbf{E}+(\boldsymbol{\mu}+(\mathbf{m} \cdot \nabla) \boldsymbol{\mu}) \mathbf{H} \cdot \mathbf{H} \mathrm{d} \mathbf{x} \mathrm{d} t \\
= & -\frac{1}{2} \int_{0}^{T} \int_{\Gamma} \boldsymbol{\nu} \cdot \mathbf{m}(\boldsymbol{\mu} \mathbf{H} \cdot \mathbf{H}+\varepsilon \mathbf{E} \cdot \mathbf{E}) \mathrm{d} \mathbf{x} \mathrm{d} t \\
& +\int_{0}^{T} \int_{\Gamma}(\boldsymbol{\nu} \times \mathbf{E}) \cdot(\mathbf{m} \times \varepsilon \mathbf{E}) \mathrm{d} \mathbf{x} \mathrm{d} t+\int_{0}^{T} \int_{\Gamma}(\boldsymbol{\nu} \times \mathbf{H}) \cdot(\mathbf{m} \times \boldsymbol{\mu H}) \mathrm{d} \mathbf{x} \mathrm{d} t \\
& +\int_{G}(\mathbf{m} \times \varepsilon \mathbf{E}(T)) \cdot(\boldsymbol{\mu} \mathbf{H}(T)) \mathrm{d} \mathbf{x}-\int_{G}(\mathbf{m} \times \varepsilon \mathbf{E}(0)) \cdot(\boldsymbol{\mu} \mathbf{H}(0)) \mathrm{d} \mathbf{x} .
\end{aligned}
$$


The left-hand side can be estimated using inequalities in Equation $(3.2)$ as

$$
\frac{1}{2} \int_{0}^{T} \int_{G}(\varepsilon+(\mathbf{m} \cdot \nabla) \varepsilon) \mathbf{E} \cdot \mathbf{E}+(\boldsymbol{\mu}+(\mathbf{m} \cdot \nabla) \boldsymbol{\mu}) \mathbf{H} \cdot \mathbf{H} \mathrm{d} \mathbf{x} \mathrm{d} t \geq \frac{d_{1}}{2} \int_{0}^{T} \int_{G} \varepsilon \mathbf{E} \cdot \mathbf{E}+\boldsymbol{\mu} \mathbf{H} \cdot \mathbf{H} \mathrm{d} \mathbf{x} \mathrm{d} t .
$$

From Assumption 2.1 and Equation (2.3), we get $\varepsilon \mathbf{E} \cdot \mathbf{E} \geq \alpha|\mathbf{E}|^{2}, \boldsymbol{\mu} \mathbf{E} \cdot \mathbf{E} \geq \alpha|\mathbf{E}|^{2}$ for all $E \in \mathbb{R}^{3}$. Therefore,

$$
\begin{aligned}
\frac{1}{2} \int_{0}^{T} \int_{G}(\varepsilon+(\mathbf{m} \cdot \nabla) \varepsilon) \mathbf{E} \cdot \mathbf{E} & +(\boldsymbol{\mu}+(\mathbf{m} \cdot \nabla) \boldsymbol{\mu}) \mathbf{H} \cdot \mathbf{H} \mathrm{d} \mathbf{x} \mathrm{d} t \\
& \geq \frac{d_{1} \alpha}{2} \int_{0}^{T} \int_{G}|\mathbf{E}|^{2}+|\mathbf{H}|^{2} \mathrm{~d} \mathbf{x} \mathrm{d} t .
\end{aligned}
$$

From the compactness of $\Gamma$ and the continuity of $\mathbf{m}$, we get $\mathbf{m} \cdot \boldsymbol{\nu} \geq \beta>0$ uniformly on $\Gamma$. Thus, the first term on the right-hand side of Equation 3.13 can be estimated via

$$
\begin{aligned}
-\frac{1}{2} \int_{0}^{T} \int_{\Gamma} \boldsymbol{\nu} \cdot \mathbf{m}(\boldsymbol{\mu} \mathbf{H} \cdot \mathbf{H}+\varepsilon \mathbf{E} \cdot \mathbf{E}) \mathrm{d} \mathbf{x} \mathrm{d} t & \leq-\frac{1}{2} \int_{0}^{T} \int_{\Gamma} \boldsymbol{\nu} \cdot \mathbf{m}(\boldsymbol{\mu} \mathbf{H} \cdot \mathbf{H}+\boldsymbol{\varepsilon} \mathbf{E} \cdot \mathbf{E}) \mathrm{d} \mathbf{x} \mathrm{d} t \\
& \leq-\frac{\beta}{2} \int_{0}^{T} \int_{\Gamma} \boldsymbol{\mu} \mathbf{H} \cdot \mathbf{H}+\boldsymbol{\varepsilon} \mathbf{E} \cdot \mathbf{E} \mathrm{d} \mathbf{x} \mathrm{d} t
\end{aligned}
$$

Utilizing Young's inequality, we further get

$$
\begin{aligned}
\mid \int_{G}(\mathbf{m} \times \boldsymbol{\varepsilon} \mathbf{E}(T)) & \cdot(\boldsymbol{\mu} \mathbf{H}(T)) \mathrm{d} \mathbf{x}\left|\leq \int_{G}\right| \mathbf{m}|\cdot| \varepsilon \mathbf{E}(T)|\cdot| \boldsymbol{\mu H}(T) \mid \mathrm{d} \mathbf{x} \\
& \leq \sup _{\mathbf{x} \in G}|\mathbf{m}(\mathbf{x})|\left|\lambda_{\max }(\varepsilon) \lambda_{\max }(\boldsymbol{\mu})\right| \int_{G}|\mathbf{E}(T)| \cdot|\mathbf{H}(T)| \mathrm{d} \mathbf{x} \\
& \leq \frac{1}{2} \sup _{\mathbf{x} \in G}|\mathbf{m}(\mathbf{x})|\left|\lambda_{\max }(\boldsymbol{\varepsilon}) \lambda_{\max }(\boldsymbol{\mu})\right| \int_{G}|\mathbf{E}(T)|^{2}+|\mathbf{H}(T)|^{2} \mathrm{~d} \mathbf{x} \\
& \leq \sup _{\mathbf{x} \in G}|\mathbf{m}(\mathbf{x})|\left|\lambda_{\max }(\boldsymbol{\varepsilon}) \lambda_{\max }(\boldsymbol{\mu})\right| E_{\xi}(T) .
\end{aligned}
$$

Similarly, we obtain

$$
\left|\int_{G}(\mathbf{m} \times \varepsilon \mathbf{E}(0)) \cdot(\boldsymbol{\mu} \mathbf{H}(0)) \mathrm{d} \mathbf{x}\right| \leq \sup _{\mathbf{x} \in G}|\mathbf{m}(\mathbf{x})|\left|\lambda_{\max }(\varepsilon) \lambda_{\max }(\boldsymbol{\mu})\right| E_{\xi}(0) .
$$

Next, we estimate $\int_{0}^{T} \int_{\Gamma}(\boldsymbol{\nu} \times \mathbf{E}) \cdot(\mathbf{m} \times \varepsilon \mathbf{E}) \mathrm{d} \mathbf{x} \mathrm{d} t$. By virtue of Young's inequality, we get

$$
|(\boldsymbol{\nu} \times \mathbf{E}) \cdot(\mathbf{m} \times \varepsilon \mathbf{E})| \leq|\boldsymbol{\nu} \times \mathbf{E}| \cdot|\mathbf{m} \times \varepsilon \mathbf{E}| \leq \frac{1}{2 \delta}|\boldsymbol{\nu} \times \mathbf{E}|^{2}+\frac{\delta}{2}|\mathbf{m} \times \varepsilon \mathbf{E}|^{2}
$$

Using the uniform positive definiteness of $\varepsilon$, we further find

$$
\begin{aligned}
|\mathbf{m} \times \varepsilon \mathbf{E}|^{2} & \leq \sup _{\mathbf{x} \in G}|\mathbf{m}(\mathbf{x})|^{2} \cdot|\varepsilon \mathbf{E}|^{2} \leq \sup _{\mathbf{x} \in G}|\mathbf{m}(\mathbf{x})|^{2}\left(\lambda_{\max }(\varepsilon)|\mathbf{E}|\right)^{2} \\
& \leq \sup _{\mathbf{x} \in G}|\mathbf{m}(\mathbf{x})|^{2}\left(\lambda_{\max }(\varepsilon)\right)^{2} \frac{1}{\alpha} \varepsilon \mathbf{E} \cdot \mathbf{E} .
\end{aligned}
$$

Integrating the latter inequality, we get

$$
\int_{0}^{T} \int_{\Gamma}|\mathbf{m} \times \varepsilon \mathbf{E}|^{2} \mathrm{~d} \mathbf{x} \mathrm{d} t \leq \frac{1}{\alpha} \sup _{\mathbf{x} \in G}|\mathbf{m}(\mathbf{x})|^{2}\left(\lambda_{\max }(\varepsilon)\right)^{2} \int_{0}^{T} \int_{\Gamma} \varepsilon \mathbf{E} \cdot \mathbf{E} \mathrm{d} \mathbf{x} \mathrm{d} t .
$$


Using Equations (3.18) and 3.19, we obtain

$$
\begin{aligned}
\int_{0}^{T} \int_{\Gamma}(\boldsymbol{\nu} \times \mathbf{E}) \cdot(\mathbf{m} \times \varepsilon \mathbf{E}) \mathrm{d} \mathbf{x} \mathrm{d} t & \leq \int_{0}^{T} \int_{\Gamma} \frac{1}{2 \delta}|\boldsymbol{\nu} \times \mathbf{E}|^{2}+\frac{\delta}{2}|\mathbf{m} \times \varepsilon \mathbf{E}|^{2} \mathrm{~d} \mathbf{x} \mathrm{d} t \\
& \leq \frac{1}{2 \delta} \int_{0}^{T} \int_{\Gamma}|\boldsymbol{\nu} \times \mathbf{E}|^{2} \mathrm{~d} \mathbf{x} \mathrm{d} t \\
& +\frac{\delta}{2} \frac{1}{\alpha} \sup _{\mathbf{x} \in G}|\mathbf{m}(\mathbf{x})|^{2}\left(\lambda_{\max }(\varepsilon)\right)^{2} \int_{0}^{T} \int_{\Gamma} \varepsilon \mathbf{E} \cdot \mathbf{E} \mathrm{d} \mathbf{x} \mathrm{d} t .
\end{aligned}
$$

In the same fashion, we get

$$
\begin{aligned}
\int_{0}^{T} \int_{\Gamma}(\boldsymbol{\nu} \times \mathbf{H}) \cdot(\mathbf{m} \times \boldsymbol{\mu H}) \mathrm{d} \mathbf{x} \mathrm{d} t & \leq \int_{0}^{T} \int_{\Gamma} \frac{1}{2 \delta}|\boldsymbol{\nu} \times \mathbf{H}|^{2}+\frac{\delta}{2}|\mathbf{m} \times \boldsymbol{\mu} \mathbf{H}|^{2} \mathrm{~d} \mathbf{x} \mathrm{d} t \\
& \leq \frac{1}{2 \delta} \int_{0}^{T} \int_{\Gamma}|\boldsymbol{\nu} \times \mathbf{H}|^{2} \mathrm{~d} \mathbf{x} \mathrm{d} t \\
& +\frac{\delta}{2} \frac{1}{\alpha} \sup _{\mathbf{x} \in G}|\mathbf{m}(\mathbf{x})|^{2}\left(\lambda_{\max }(\boldsymbol{\mu})\right)^{2} \int_{0}^{T} \int_{\Gamma} \boldsymbol{\mu} \mathbf{H} \cdot \mathbf{H} \mathrm{d} \mathbf{x} \mathrm{d} t .
\end{aligned}
$$

Recalling the boundary condition in Equation (1.11), we estimate

$$
\begin{aligned}
\frac{1}{2 \delta} \int_{0}^{T} \int_{\Gamma}|\boldsymbol{\nu} \times \mathbf{H}|^{2} \mathrm{~d} \mathbf{x} \mathrm{d} t & =\frac{1}{2 \delta} \int_{0}^{T} \int_{\Gamma}\left|\left(\gamma_{1} \mathbf{g}\left(\left.\mathbf{Z}\right|_{s=0}\right)+\gamma_{2} \mathbf{g}\left(\left.\mathbf{Z}\right|_{s=1}\right)\right) \times \boldsymbol{\nu}\right|^{2} \mathrm{~d} \mathbf{x} \mathrm{d} t \\
& \leq \frac{1}{2 \delta} \int_{0}^{T} \int_{\Gamma}\left|\left(\gamma_{1} \mathbf{g}\left(\left.\mathbf{Z}\right|_{s=0}\right)+\gamma_{2} \mathbf{g}\left(\left.\mathbf{Z}\right|_{s=1}\right)\right)\right|^{2} \mathrm{~d} \mathbf{x} \mathrm{d} t \\
& \leq \frac{\max \left\{\gamma_{1}^{2}, \gamma_{2}^{2}\right\}}{\delta} \int_{0}^{T} \int_{\Gamma} \mathbf{g}^{2}\left(\left.\mathbf{Z}\right|_{s=0}\right)+\mathbf{g}^{2}\left(\left.\mathbf{Z}\right|_{s=1}\right) \mathrm{d} \mathbf{x} \mathrm{d} t \\
& \leq\left.\frac{c_{2}^{2} \max \left\{\gamma_{1}^{2}, \gamma_{2}^{2}\right\}}{\delta} \int_{0}^{T} \int_{\Gamma} \mathbf{Z}\right|_{s=0} ^{2}+\left.\mathbf{Z}\right|_{s=1} ^{2} \mathrm{~d} \mathbf{x} \mathrm{d} t
\end{aligned}
$$

Combining Equations (3.13)-(3.17) and (3.20)-(3.22), we deduce

$$
\begin{aligned}
\frac{d_{1} \alpha}{2} \int_{0}^{T} \int_{G}|\mathbf{E}|^{2}+|\mathbf{H}|^{2} \mathrm{~d} \mathbf{x} \mathrm{d} t \leq & -\frac{\beta}{2} \int_{0}^{T} \int_{\Gamma} \boldsymbol{\mu H} \cdot \mathbf{H}+\boldsymbol{\varepsilon} \mathbf{E} \cdot \mathbf{E} \mathrm{d} \mathbf{x} \mathrm{d} t \\
& +\frac{1}{2 \delta} \int_{0}^{T} \int_{\Gamma}|\boldsymbol{\nu} \times \mathbf{E}|^{2} \mathrm{~d} \mathbf{x} \mathrm{d} t \\
& +\frac{\delta}{2} \cdot \frac{1}{\alpha} \sup _{\mathbf{x} \in G}|\mathbf{m}(\mathbf{x})|^{2}\left(\lambda_{\max }(\boldsymbol{\varepsilon})\right)^{2} \int_{0}^{T} \int_{\Gamma} \varepsilon \mathbf{E} \cdot \mathbf{E} \mathrm{d} \mathbf{x} \mathrm{d} t \\
& +\left.\frac{c_{2}^{2} \max \left\{\gamma_{1}^{2}, \gamma_{2}^{2}\right\}}{\delta} \int_{0}^{T} \int_{\Gamma} \mathbf{Z}\right|_{s=0} ^{2}+\left.\mathbf{Z}\right|_{s=1} ^{2} \mathrm{~d} \mathbf{x} \mathrm{d} t \\
& +\frac{\delta}{2} \frac{1}{\alpha} \sup _{\mathbf{x} \in G}|\mathbf{m}(\mathbf{x})|^{2}\left(\lambda_{\max }(\boldsymbol{\mu})\right)^{2} \int_{0}^{T} \int_{\Gamma} \boldsymbol{\mu H} \cdot \mathbf{H} \mathrm{d} \mathbf{x} \mathrm{d} t \\
& +\sup _{\mathbf{x} \in G}|\mathbf{m}(\mathbf{x})|\left|\lambda_{\max }(\boldsymbol{\varepsilon}) \lambda_{\max }(\boldsymbol{\mu})\right|\left(E_{\xi}(T)+E_{\xi}(0)\right) .
\end{aligned}
$$

Choosing $\delta>0$ such that

$$
\frac{\delta}{2} \frac{1}{\alpha} \sup _{\mathbf{x} \in G}|\mathbf{m}(\mathbf{x})|^{2} \max \left\{\left(\lambda_{\max }(\varepsilon)\right)^{2},\left(\lambda_{\max }(\boldsymbol{\mu})\right)^{2}\right\} \leq \frac{\beta}{2},
$$


we arrive at

$$
\begin{aligned}
\frac{d_{1} \alpha}{2} \int_{0}^{T} \int_{G}|\mathbf{E}|^{2}+|\mathbf{H}|^{2} \mathrm{~d} \mathbf{x} \mathrm{d} t \leq & \left.\frac{1}{2 \delta} \int_{0}^{T} \int_{\Gamma} \mathbf{Z}\right|_{s=0} ^{2} \mathrm{~d} \mathbf{x} \mathrm{d} t \\
& +\left.\frac{c_{2}^{2} \max \left\{\gamma_{1}^{2}, \gamma_{2}^{2}\right\}}{\delta} \int_{0}^{T} \int_{\Gamma} \mathbf{Z}\right|_{s=0} ^{2}+\left.\mathbf{Z}\right|_{s=1} ^{2} \mathrm{~d} \mathbf{x} \mathrm{d} t \\
& +\sup _{\mathbf{x} \in G}|\mathbf{m}(\mathbf{x})|\left|\lambda_{\max }(\varepsilon) \lambda_{\max }(\boldsymbol{\mu})\right|\left(E_{\xi}(T)+E_{\xi}(0)\right) .
\end{aligned}
$$

There remains to estimate

$$
I=\int_{0}^{T} \int_{0}^{1} \int_{\Gamma}|\mathbf{E}(t-\tau s, \mathbf{x}) \times \boldsymbol{\nu}|^{2} \mathrm{~d} \mathbf{x} \mathrm{d} s \mathrm{~d} t .
$$

Making substitution $u=t-\tau s$ and $v=t$, we get

$$
\begin{aligned}
I & =\frac{1}{\tau} \int_{0}^{T} \int_{v-\tau}^{v} \int_{\Gamma}|\mathbf{E}(u, \mathbf{x}) \times \boldsymbol{\nu}|^{2} \mathrm{~d} \mathbf{x} \mathrm{d} s \mathrm{~d} v \\
& =\frac{1}{\tau} \int_{-\tau}^{0}(u+\tau) \int_{\Gamma}|\mathbf{E}(u, \mathbf{x}) \times \boldsymbol{\nu}|^{2} \mathrm{~d} \mathbf{x} \mathrm{d} u+\frac{1}{\tau} \int_{0}^{T-\tau} \tau \int_{\Gamma}|\mathbf{E}(u, \mathbf{x}) \times \boldsymbol{\nu}|^{2} \mathrm{~d} \mathbf{x} \mathrm{d} u \\
& +\frac{1}{\tau} \int_{T-\tau}^{T}(T-u) \int_{\Gamma}|\mathbf{E}(u, \mathbf{x}) \times \boldsymbol{\nu}|^{2} \mathrm{~d} \mathbf{x} \mathrm{d} u \\
& \leq \int_{-\tau}^{0} \int_{\Gamma}|\mathbf{E}(u, \mathbf{x}) \times \boldsymbol{\nu}|^{2} \mathrm{~d} \mathbf{x} \mathrm{d} u+\int_{0}^{T-\tau} \int_{\Gamma}|\mathbf{E}(u, \mathbf{x}) \times \boldsymbol{\nu}|^{2} \mathrm{~d} \mathbf{x} \mathrm{d} u \\
& +\int_{T-\tau}^{T} \int_{\Gamma}|\mathbf{E}(u, \mathbf{x}) \times \boldsymbol{\nu}|^{2} \mathrm{~d} \mathbf{x} \mathrm{d} u \\
& \leq \int_{0}^{T} \int_{\Gamma}|\mathbf{E}(u, \mathbf{x}) \times \boldsymbol{\nu}|^{2} \mathrm{~d} \mathbf{x} \mathrm{d} u+\int_{0}^{T} \int_{\Gamma}|\mathbf{E}(u-\tau, \mathbf{x}) \times \boldsymbol{\nu}|^{2} \mathrm{~d} \mathbf{x} \mathrm{d} u \\
& =\left.\int_{0}^{T} \int_{\Gamma} \mathbf{Z}\right|_{s=0} ^{2}+\left.\mathbf{Z}\right|_{s=1} ^{2} \mathrm{~d} \mathbf{x} \mathrm{d} t
\end{aligned}
$$

Now, multiplying Equation (3.25) by $\xi \tau$ and adding the result to Equation (3.24) divided by $d_{1} \alpha$, the claim follows with appropriate constants $c, c_{T}$.

Theorem 3.5. Let $\mathbf{V}$ be the unique strong solution given in Theorem 2.8. Under Assumption 3.1, if $c_{1} \gamma_{1}>c_{2} \gamma_{2}$ (i.e., the delay term is not too strong), there exist $C, \lambda>0$ such that the associated energy satisfies

$$
E(t) \leq C e^{-\lambda t} E(0) \text { for } t \geq 0 .
$$

Proof. From Lemmas 3.3, 3.4 and Theorem A.1 in the appendix with

$$
D(t)=\left.\int_{\Gamma} \mathbf{Z}\right|_{s=0} ^{2}+\left.\mathbf{Z}\right|_{s=1} ^{2} \mathrm{~d} \mathbf{x}
$$

we get the desired inequality for $E_{\xi}(\cdot)$ in place of $E(\cdot)$. Taking into account the equivalence of $E(\cdot)$ and $E_{\xi}(\cdot)$, the original claim follows.

Due to the density of $D(\mathscr{A})$ in $\mathscr{H}$, we have:

Corollary 3.6. The conclusions of Theorem 3.5 remain true for mild solutions, i.e., if $\mathbf{V}^{0} \in \mathscr{H}$. 


\section{Acknowledgment}

Financial support by the Deutsche Forschungsgemeinschaft (DFG) - through CRC 1173 at Karlsruhe Institute of Technology, Germany - and the University of Texas at El Paso is gratefully acknowledged.

\section{References}

[1] V. Barbu. Nonlinear Differential Equations of Monotone Types in Banach Spaces. Springer Monographs in Mathematics. Springer-Verlag, NY, 2010.

[2] J. Cagnol and M. Eller. Boundary regularity for Maxwell's equations with applications to shape optimization. Journal of Differential Equations, 250:1114-1136, 2011.

[3] F. Cakoni, D. Colton, and P. Monk. The Linear Sampling Method in Inverse Electromagnetic Scattering, volume 80 of CBMS-NSF Regional Conference Series in Applied Mathematics. SIAM, Philadelphia, PA, 2011.

[4] R. Datko. Two examples of ill-posedness with respect to time delays revisited. IEEE Trans. Automatic Control, 42:511-515, 1997.

[5] R. Datko, J. Lagnese, and M.P. Polis. An example of the effect of time delays in boundary feedback stabilization of wave equations. SIAM Journal of Control and Optimization, 24(1):152-156, 1986.

[6] M. Eller. Continuous observability for the anisotropic Maxwell system. Applied Mathematics ES Optimization, 55:185-201, 2007.

[7] M. Eller. On boundary regularity of solutions to Maxwell's equations with a homogeneous conservative boundary condition. Discrete and Continuous Dynamical Systems: Series S, 2(3):473481, 2009.

[8] M. Eller, J. Lagnese, and S. Nicaise. Decay rates for solutions of a Maxwell system with nonlinear boundary damping. Computational and Applied Mathematics, 21(1):135-165, 2002.

[9] M. Eller, J.E. Lagnese, and S. Nicaise. Stabilization of heterogeneous Maxwell's equations by linear and nonlinear boundary feedbacks. Electronic Journal of Differential Equations, 21:1-26, 2002.

[10] M. Eller and J.E. Masters. Exact boundary controllability of electromagnetic fields in a general region. Applied Mathematics \&S Optimization, 45:99-123, 2002.

[11] F. Jochmann. Exponential decay of solutions of Maxwell's equations coupled with a firstorder ordinary differential equation for the polarization. Journal of Mathematical Analysis and Applications, 288:411-423, 2003.

[12] B.V. Kapitonov. Stabilization and exact boundary controllability for Maxwell's equations. SIAM Journal of Control and Optimization, 32:408-420, 1994.

[13] D.Ya. Khusainov, M. Pokojovy, and R. Racke. Strong and mild extrapolated $L^{2}$-solutions to the heat equation with constant delay. SIAM Journal of Mathematical Analysis, 47(1):427-454, 2015 . 
[14] V. Komornik. Boundary stabilization, observation and control of Maxwell's equations. PanAmerican Mathematical Journal, 4:47-61, 1994.

[15] S.S. Krigman. Exact boundary controllability of Maxwell's equations with weak conductivity in the heterogeneous medium inside a general domain. Discrete and Continuous Dynamical Systems, 2007:590-601, 2007.

[16] J. Lagnese. Exact boundary controllability of Maxwell's equations in a general region. SIAM Journal of Control and Optimization, 27:374-388, 1989.

[17] I. Lasiecka and D. Tataru. Uniform boundary stabilization of semilinear wave equations with nonlinear boundary damping. Differential and Integral Equations, 6(3):507-533, 51993.

[18] R. Nibbi and S. Polidoro. Exponential decay for Maxwell equations with a boundary memory condition. Journal of Mathematical Analysis and Applications, 302:30-55, 2005.

[19] S. Nicaise. Exact boundary controllability of Maxwell's equations in heterogeneous media and and application to an inverse source problem. SIAM Journal of Control and Optimization, 38(4):1145-1170, 2000.

[20] S. Nicaise and C. Pignotti. Boundary stabilization of Maxwell's equations with space-time variable coefficients. ESAIM: Control, Optimisation and Calculus of Variations, 9:563-578, August 2003.

[21] S. Nicaise and C. Pignotti. Internal stabilization of Maxwell's equations in heterogeneous media. Abstract and Applied Analysis, 2005(7):791-811, 2005.

[22] S. Nicaise and C. Pignotti. Internal and boundary observability estimates for heterogeneous Maxwell's system. Applied Mathematics \& Optimization, 54:47-70, 2006.

[23] S. Nicaise and C. Pignotti. Stability and instability results of the wave equation with a delay term in the boundary or internal feedbacks. SIAM Journal of Control and Optimization, 45(5):1561-1585, 2006.

[24] R.E. Showalter. Monotone Operators in Banach Space and Nonlinear Partial Differential Equations, volume 49 of Mathematical Surveys and Monographs. AMS, Providence, RI, 1997.

[25] L. Zhang and G. Stepan. Stability analysis of the wave equation with delayed boundary conditions. IUTAM Symposium on Nonlinear and Delayed Dynamics of Mechatronic Systems, 22:139-145, 2017.

[26] X. Zhang. Exact internal controllability of Maxwell's equations. Applied Mathematics 83 Optimization, 41:155-170, 2000.

[27] Q. Zhou. Exact internal controllability of Maxwell's equations. Japan Journal of Industrial and Applied Mathematics, 14:245-256, 1997.

\section{A Proof of Exponential Stability}

Theorem A.1. Suppose there exist a non-negative function $D(t)$ and positive numbers $c_{1}^{E}, c_{2}^{E}, c$ and $c_{T}$ such that

$$
-c_{1}^{E} \int_{t_{1}}^{t_{2}} D(t) \mathrm{d} t \geq E\left(t_{2}\right)-E\left(t_{1}\right) \geq-c_{2}^{E} \int_{t_{1}}^{t_{2}} D(t) \mathrm{d} t \text { for all } t_{2}>t_{1} \geq 0
$$


and

$$
\int_{0}^{T} E(t) \mathrm{d} t \leq c(E(0)+E(T))+c_{T} \int_{0}^{T} D(t) \mathrm{d} t \text { for arbitrarily large } T .
$$

Then, there exist $C, \lambda>0$ such that the function $E(t)$ satisfies

$$
E(t) \leq C e^{-\lambda t} E(0) \text { for } t \geq 0 .
$$

Proof. Taking $t_{1}=0$ and $t_{2}=T$ in Equation A.1, we get

$$
E(0) \leq E(T)+c_{2}^{E} \int_{0}^{T} D(t) \mathrm{d} t .
$$

Thus, from Equation A.2, we obtain

$$
\int_{0}^{T} E(t) \mathrm{d} t \leq 2 c E(T)+\left(c_{T}+c c_{2}^{E}\right) \int_{0}^{T} D(t) \mathrm{d} t .
$$

Now, using Equation A.1 with $t_{2}=T$ and $t_{1}=t$, we get

$$
E(t) \geq E(T)+c_{1}^{E} \int_{t}^{T} D(s) \mathrm{d} s .
$$

Integrating the latter inequality from 0 to $T$ with respect to $t$ and taking into account Equation A.4, we arrive at

$$
T E(T)+c_{1}^{E} \int_{0}^{T} \int_{t}^{T} D(s) \mathrm{d} s \mathrm{~d} t \leq \int_{0}^{T} E(t) \mathrm{d} t \leq 2 c E(T)+\left(c_{T}+c c_{2}^{E}\right) \int_{0}^{T} D(t) \mathrm{d} t .
$$

Choosing $T>4 c$, we have

$$
\frac{T}{2} E(T)+c_{1}^{E} \int_{0}^{T} \int_{t}^{T} D(s) \mathrm{d} s \mathrm{~d} t \leq\left(c_{T}+c c_{2}^{E}\right) \int_{0}^{T} D(t) \mathrm{d} t .
$$

Since $D(s)$ is non-negative, we estimate

$$
\frac{T}{2} E(T) \leq\left(c_{T}+c c_{2}^{E}\right) \int_{0}^{T} D(t) \mathrm{d} t .
$$

Applying Equation A.1 with $t_{1}=0$ and $t_{2}=T$ to the inequality in Equation (A.8), we get

$$
\frac{T}{2} E(T) \leq \frac{c_{T}+c c_{2}^{E}}{c_{1}^{E}}(E(0)-E(T))
$$

which finally leads us to

$$
\left(\frac{T}{2}+\tilde{c}\right) E(T) \leq \tilde{c} E(0)
$$

with $\tilde{c}=\frac{c_{T}+c c_{2}^{E}}{c_{1}^{E}}$. Thus,

$$
E(T) \leq \gamma E(0) \text { for } \gamma=\frac{\tilde{c}}{\tilde{c}+T / 2}<1 .
$$

Using a similar argument on each of the time segments $[(m-1) T, m T]$ for $m=1,2, \ldots$, we obtain

$$
E(m T) \leq \gamma E((m-1) T) \leq \ldots \leq \gamma^{m} E(0), m=1,2, \ldots
$$


Denoting $\lambda=-T^{-1} \ln (\gamma)>0$, Equation A.12 rewrites as

$$
E(m T) \leq e^{-\lambda m T} E(0), m=1,2, \ldots
$$

It easily follows from A.1 that $E(t)$ is monotone non-increasing. This leads to

$$
E(t) \leq E(m T) \leq e^{-\lambda m T} E(0)=\frac{1}{\gamma} e^{-\lambda(m+1) T} E(0) \leq \frac{1}{\gamma} e^{-\lambda t} E(0)
$$

for arbitrary $t \in[m T,(m+1) T]$ for any $m=1,2, \ldots$, which completes the proof. 